\title{
New Practical Integral Variable Structure Controllers for Uncertain Nonlinear Systems
}

\author{
Jung-Hoon Lee \\ Gyeongsang National University \\ South Korea
}

\section{Introduction}

Stability analysis and controller design for uncertain nonlinear systems is open problem now(Vidyasagar, 1986). So far numerous design methodologies exist for the controller design of nonlinear systems(Kokotovic \& Arcak, 2001). These include any of a huge number of linear design techniques(Anderson \& More, 1990; Horowitz, 1991) used in conjuction with gain scheduling(Rugh \& Shamma, 200); nonlinear design methodologies such as Lyapunov function approach(Vidyasagar, 1986; Kokotovic \& Arcak, 2001; Cai et al., 2008; Gutman, 1979; Slotine \& Li, 1991; Khalil, 1996), feedback linearization method(Hunt et al., 1987; Isidori, 1989; Slotine \& Li, 1991), dynamics inversion(Slotine \& Li, 1991), backstepping(Lijun \& Chengkand, 2008), adaptive technique which encompass both linear adaptive(Narendra, 1994) and nonlinear adaptive control(Zheng \& Wu, 2009), sliding mode control(SMC)(Utkin, 1978; Decarlo etal., 1988; Young et al., 1996; Drazenovic, 1969; Toledo \& Linares, 1995; Bartolini \& Ferrara, 1995; Lu \& Spurgeon, 1997), and etc(Hu \& Martin, 1999; Sun, 2009; Chen, 2003).

The sliding mode control can provide the effective means to the problem of controlling uncertain nonlinear systems under parameter variations and external disturbances(Utkin, 1978; Decarlo et. al., 1988; Young et al., 1996). One of its essential advantages is the robustness of the controlled system to variations of parameters and external disturbances in the sliding mode on the predetermined sliding surface, $s=0$ (Drazenovic, 1969). In the VSS, there are the two main problems, i.e., the reaching phase at the initial stage(Lee \& Youn, 1994) and chattering of the input (Chern \& Wu, 1992). To remove the reaching phase, the two requirements are needed, i.e., the sliding surface must be determined from an any given initial state to the origin $\left(\left.s(x)\right|_{x=x(0) \quad \& \mathrm{t}=0}=0\right)$ and the control input must satisfy the existence condition of the sliding mode on the pre-selected sliding surface for all time from the initial to the final time $\left(s^{T} \dot{s}<0\right.$, for $\left.t \geq 0\right)$.

In (Toledo \& Linares, 1995), the sliding mode approach is applied to nonlinear output regulator schemes. The underlying concept is that of designing sliding submanifold which contains the zero tracking error sub-manifold. The convergence to a sliding manifold can be attained relying on a control strategy based on a simplex of control vectors for multi input uncertain nonlinear systems(Bartolini \& Ferrara, 1995). A nonlinear optimal integral variable 
structure controller with an arbitrary sliding surface without the reaching phase was proposed for uncertain linear plants(Lee, 1995). (Lu and Spurgeon, 1997) considered the robustness of dynamic sliding mode control of nonlinear system, which is in differential input-output form with additive uncertainties in the model. The discrete-time implementation of a second-order sliding mode control scheme is analyzed for uncertain nonlinear system in (Bartolini et al., 2001). (Adamy \& Flemming, 2004) surveyed so called soft variable structure controls, compared them to others. The tracker control problem that is the regulation control problem from an arbitrary initial state to an arbitrary final state without the reaching phase is handled and solved for uncertain SISO linear plants in (Lee, 2004). For 2 nd order uncertain nonlinear system with mismatched uncertainties, a switching control law between a first order sliding mode control and a second order sliding mode control is proposed to obtain the globally or locally asymptotic stability(Wang et al., 2007). The optimal SMC for nonlinear system with time-delay is suggested(Tang et al., 2008). The nonlinear time varying sliding sector is designed for continuous control of a single input nonlinear time varying input affine system which can be represented in the form of state dependent linear time variant systems with matched uncertainties(Pan et al., 2009). For uncertain affine nonlinear systems with mismatched uncertainties and matched disturbance, the systematic design of the SMC is reported(Lee, 2010a). The two clear proofs of the existence condition of the sliding mode with respect to the two transformations i.e., the two diagonalization methods are given for multi-input uncertain linear plants(Lee 2010b), while (Utkin, 1978) and (Decarlo et al., 1988) proved unclearly for uncertain nonlinear plants.

Until now, the integral action is not introduced to the variable structure system for uncertain nonlinear system with mismatched uncertainties and matched disturbance to improve the output performance by means of removing the reaching phase problems. And a nonlinear output feedback controller design for uncertain nonlinear systems with mismatched uncertainties and matched disturbance is not presented.

In this chapter, a systematic general design of new integral nonlinear full-state(output) feedback variable structure controllers based on state dependent nonlinear form is presented for the control of uncertain affine nonlinear systems with mismatched uncertainties and matched disturbances. After an affine uncertain nonlinear system is represented in the form of state dependent nonlinear system, a systematic design of a new nonlinear full-state(output) feedback variable structure controller is presented. To be linear in the closed loop resultant dynamics, full-state(output) feedback (transformed) integral linear sliding surfaces are applied in order to remove the reaching phase, those are stemmed from the studys by (Lee \& Youn, 1994; Lee, 2010b) which is the first time work of removing the reaching phase with the idea of introducing the initial condition for the integral state. The corresponding discontinuous (transformed) control inputs are proposed to satisfy the closed loop exponential stability and the existence condition of the sliding mode on the fullstate(output) feedback integral sliding surfaces, which will be investigated in Theorem 1 and Theorem 2. For practical application to the real plant by means of removing the chattering problems, the implementation of the continuous approximation is essentially needed instead of the discontinuous input as the inherent property of the VSS. Using the saturation function, the different form from that of (Chern \& Wu, 1992) for the continuous approximation is suggested. The two main problems of the VSS are removed and solved. Through the design examples and simulation studies, the usefulness of the proposed practical integral nonlinear VSS controller is verified. 


\section{Practical integral nonlinear variable structure systems}

\subsection{Descriptions of plants}

Consider an affine uncertain nonlinear system

$$
\begin{gathered}
\dot{x}=f^{\prime}(x, t)+g(x, t) u+\bar{d}(x, t), \quad x(0) \\
y=C \cdot x, \quad y(0)=C \cdot x(0)
\end{gathered}
$$

where $x \in R^{n}$ is the state, $x(0)$ is its initial condition for the state, $y \in R^{q}, q \leq n$ is the output, $y(0)$ is an initial condition of the output, $u \in R^{1}$ is the control to be determined, mismatched uncertainty $f^{\prime}(x, t) \in C^{k}$ and matched uncertainty $g(x, t) \in C^{k}, k \geq 1$, $g(x, t) \neq 0$ for all $x \in R^{n}$ and for all $t \geq 0$ are of suitable dimensions, and $\bar{d}(x, t)$ implies bounded matched external disturbances.

Assumption (Pan et al., 2009)

A1: $f^{\prime}(x, t) \in C^{k}$ is continuously differentiable and $f^{\prime}(0, t)=0$ for all $t \geq 0$.

Then, uncertain nonlinear system (1) can be represented in more affine nonlinear system of state dependent coefficient form(Pan et al., 2009; Hu \& Martin, 1999; Sun, 2009)

$$
\begin{gathered}
\dot{x}=f(x, t) x+g(x, t) u+\bar{d}(x, t), \quad x(0) \\
=\left[f_{0}(x, t)+\Delta f(x, t)\right] x+\left[g_{0}(x, t)+\Delta g(x, t)\right] u+\bar{d}(x, t) \\
=f_{0}(x, t) x+g_{0}(x, t) u+d(x, t) \\
y=C \cdot x, \\
d(x, t)=\Delta f(x, t) x+\Delta g(x, t) u+\bar{d}(x, t)
\end{gathered}
$$

where $f_{0}(x, t)$ and $g_{0}(x, t)$ is each nominal value such that $f^{\prime}(x, t)=\left[f_{0}(x, t)+\Delta f(x, t)\right] x$ and $g(x, t)=\left[g_{0}(x, t)+\Delta g(x, t)\right]$, respectively, $\Delta f(x, t)$ and $\Delta g(x, t)$ are mismatched or matched uncertainties, and $d(x, t)$ is the mismatched lumped uncertainty.

Assumption:

A2: The pair $\left(f_{0}(x, t), g_{0}(x, t)\right)$ is controllable and $\left(f_{0}(x, t), C\right)$ is observable for all $x \in R^{n}$ and all $t \geq 0$ (Sun, 2009).

A3: The lumped uncertainty $d(x, t)$ is bounded.

A4: $\ddot{x}$ is bounded if $\dot{u}$ and $\dot{d}(x, t)$ is bounded.

\subsection{Full sate feedback practical integral variable structure controller}

\subsubsection{Full-state feedback integral sliding surface}

For use later, the integral term of the full-state is augmented as

$$
x_{0}=\int_{0}^{t} x(\tau) d \tau+\int_{-\infty}^{0} x(\tau) d \tau=\int_{0}^{t} x(\tau) d \tau+x_{0}(0)
$$

To control uncertain nonlinear system (1) or (3) with a linear closed loop dynamics and without reaching phase, the full-state feedback integral sliding surface used in this design is as follows: 


$$
S_{f}=L_{1} x+L_{1} x_{0}=\left[\begin{array}{ll}
L_{1} & L_{0}
\end{array}\right] \cdot\left[\begin{array}{l}
x \\
x_{0}
\end{array}\right](=0)
$$

where

$$
x_{0}(0)=-L_{0}^{-} L_{1} x(0)
$$

and $L_{0}^{-}=\left(L_{0}^{T} W L_{0}\right)^{-1} L_{0}^{T} W$, which is stemmed from the work by (Lee \& Youn, 1994). At $t=0$, the full-state feedback integral sliding surface is zero, Hence, the one of the two requirements is satisfied. Without the initial condition of the integral state, the reaching phase is not removed except the exact initial state on the sliding surface. With the initial condition (7a) for the integral state, the work on removing the reaching phase was reported by (Lee \& Youn, 1994) for the first time, which is applied to the VSS for uncertain linear plants. In (7), $L_{1}$ is a non zero element as the design parameter such that the following assumption is satisfied.

\section{Assumption}

A5: $L_{1} g(x, t)$ and $L_{1} g_{0}(x, t)$ have the full rank, i.e. those are invertible

A6: $L_{1} \Delta g(x, t)\left[L_{1} g_{0}(x, t)\right]^{-1}=\Delta I$ and $|\Delta I| \leq \xi<1$.

In (7), the design parameters $L_{1}$ and $L_{0}$ satisfy the following relationship

$$
\begin{gathered}
L_{1}\left[f_{0}(x, t)-g_{0}(x, t) K(x)\right]+L_{0}=0 \\
L_{0}=-L_{1}\left[f_{0}(x, t)-g_{0}(x, t) K(x)\right]=-L_{1} f_{c}(x, t) \\
f_{c}(x, t)=\left[f_{0}(x, t)-g_{0}(x, t) K(x)\right]
\end{gathered}
$$

The equivalent control input is obtained using $\dot{S}_{f}=0$ as(Decarlo et al., 1998)

$$
u_{e q}=-\left[L_{1} g(x, t)\right]^{-1}\left[L_{1} f_{0}(x, t)+L_{0}\right] x-\left[L_{1} g(x, t)\right]^{-1} \Delta f(x, t) x-\left[L_{1} g(x, t)\right]^{-1} \bar{d}(x, t)
$$

This control input can not be implemented because of the uncertainties, but used to obtaining the ideal sliding dynamics. The ideal sliding mode dynamics of the sliding surface (7) can be derived by the equivalent control approach(Lee, 2010a) as

$$
\begin{gathered}
\dot{x}_{s}=\left[f_{0}\left(x_{s}, t\right)-g_{0}\left(x_{s}, t\right)\left[L_{1} g\left(x_{s}, t\right)\right]^{-1}\left\{L_{1} f_{0}\left(x_{s}, t\right)+L_{0}\right\}\right] x_{s}, \quad x_{s}(0)=x(0) \\
\dot{x}_{s}=\left[f_{0}\left(x_{s}, t\right)-g_{0}\left(x_{s}, t\right) K\left(x_{s}\right)\right] x_{s}=f_{c}\left(x_{s}, t\right) x_{s}, \quad x_{s}(0)=x(0) \\
K\left(x_{s}\right)=\left[L_{1} g\left(x_{s}, t\right)\right]^{-1}\left\{L_{1} f_{0}\left(x_{s}, t\right)+L_{0}\right\}
\end{gathered}
$$

The solution of (10) or (11) identically defines the integral sliding surface. Hence to design the sliding surface as stable, this ideal sliding dynamics is designed to be stable, the reverse argument also holds. To choose the stable gain based on the Lyapunov stability theory, the ideal sliding dynamics (10) or (11) is represented by the nominal plant of (3) as

$$
\begin{aligned}
\dot{x} & =f_{0}(x, t) x+g_{0}(x, t) u, & & u=-K(x) x \\
& =f_{c}(x, t) x, & & f_{c}(x, t)=f_{0}(x, t)-g_{0}(x, t) K(x)
\end{aligned}
$$


To select the stable gain, take a Lyapunov function candidate as

$$
V(x)=x^{T} P x, \quad P>0
$$

The derivative of (14) becomes

$$
\dot{V}(x)=x^{T}\left[f_{0}^{T}(x, t) P+P f_{0}(x, t)\right] x+u^{T} g_{0}^{T}(x, t) P x+x^{T} P g_{0}(x, t) u
$$

By the Lyapunov control theory(Slotine \& Li, 1991), take the control input as

$$
u=-g_{0}^{T}(x, t) P x
$$

and $Q(x, t)>0$ and $Q_{c}(x, t)>0$ for all $x \in R^{n}$ and all $t \geq 0$ is

$$
\begin{aligned}
& f_{0}^{T}(x, t) P+P f_{0}(x, t)=-Q(x, t) \\
& f_{c}^{T}(x, t) P+P f_{c}(x, t)=-Q_{c}(x, t)
\end{aligned}
$$

then

$$
\begin{aligned}
\dot{V}(x) & =-x^{T} Q(x, t) x-2 x^{T} P g_{0}(x, t) g_{0}^{T}(x, t) P x \\
& =-x^{T}\left[Q(x, t)+2 P g_{0}(x, t) g_{0}^{T}(x, t) P\right] x \\
& =-x^{T}\left[f_{c}^{T}(x, t) P+P f_{c}(x, t)\right] x \\
& =-x^{T} Q_{c}(x, t) x \\
& \leq-\lambda_{\min }\left\{Q_{c}(x, t)\right\}|| x||^{2}
\end{aligned}
$$

where $\lambda_{\min }\left\{Q_{c}(x, t)\right\}$ means the minimum eigenvalue of $Q_{c}(x, t)$. Therefore the stable static nonlinear feedback gain is chosen as

$$
K(x)=g_{0}^{T}(x, t) P \quad \text { or } \quad=\left[L_{1} g_{0}(x, t)\right]^{-1}\left\{L_{1} f_{0}(x, t)+L_{0}\right\}
$$

\subsubsection{Full-state feedback transformed discontinuous control input}

The corresponding control input with the transformed gains is proposed as follows:

$$
u_{f}=-K(x) x-\Delta K x-K_{1} S_{f}-K_{2} \operatorname{sign}\left(S_{f}\right)
$$

where $K(x)$ is a static nonlinear feedback gain, $\Delta K$ is a discontinuous switching gain, $K_{1}$ is a static feedback gain of the sliding surface itself, and $K_{2}$ is a discontinuous switching gain, respectively as

$$
\begin{gathered}
\Delta K=\left[L_{1} g_{0}(x, t)\right]^{-1}\left[\Delta k_{i}\right] \quad i=1, \ldots, n \\
\Delta k_{i}= \begin{cases}\geq \frac{\max \left\{L_{1} \Delta f(x . t)-L_{1} \Delta g(x, t) K(x)\right\}_{i}}{\min \{I+\Delta I\}} & \operatorname{sign}\left(S_{f} x_{i}\right)>0 \\
\leq \frac{\min \left\{L_{1} \Delta f(x . t)-L_{1} \Delta g(x, t) K(x)\right\}_{i}}{\min \{I+\Delta I\}} & \operatorname{sign}\left(S_{f} x_{i}\right)<0\end{cases}
\end{gathered}
$$




$$
\begin{gathered}
K_{1}=\left[L_{1} g(x, t)\right]^{-1} K_{1}{ }^{\prime}, \quad K_{1}{ }^{\prime}>0 \\
K_{2}=\left[L_{1} g(x, t)\right]^{-1} K_{2}{ }^{\prime}, \quad K_{2}{ }^{\prime}=\frac{\max \left\{\left|L_{1} d(x, t)\right|\right\}}{\min \{I+\Delta I\}}
\end{gathered}
$$

which is transformed for easy proof of the existence condition of the sliding mode on the chosen sliding surface as the works of (Utkin, 1978; Decarlo et al., 1988; Lee, 2010b). The real sliding dynamics by the proposed control with the linear integral sliding surface is obtained as follows:

$$
\begin{aligned}
\dot{S}_{f}= & {\left[L_{1} \dot{x}+L_{0} x\right] } \\
= & L_{1}\left[f_{0}(x, t) x+\Delta f(x, t) x+g(x, t) u_{f}+\bar{d}(x, t)\right]+L_{0} x \\
= & L_{1}\left[f_{0}(x, t) x+\Delta f(x, t) x+g(x, t)\left\{-K(x) x-\Delta K x-K_{1} S_{f}-K_{2} \operatorname{sign}\left(S_{f}\right)\right\}+\bar{d}(x, t)\right]+L_{0} x \\
= & L_{1} f_{0}(x, t) x-L_{1} g_{0}(x, t) K(x) x+L_{0} x+L_{1} \Delta f(x, t) x-L_{1} \Delta g(x, t) K(x) x \\
& -L_{1} g(x, t) \Delta K x-L_{1} g(x, t) K_{1} S_{f}+L_{1} \bar{d}(x, t)-L_{1} g(x, t) K_{2} \operatorname{sign}\left(S_{f}\right) \\
= & L_{1} \Delta f(x, t) x-L_{1} \Delta g(x, t) K(x) x-[I+\Delta I] L_{1} g_{0}(x, t) \Delta K x-[I+\Delta I] L_{1} g_{0}(x, t) K_{1} S_{f} \\
& +L_{1} \bar{d}(x, t)-[I+\Delta I] L_{1} g_{0}(x, t) K_{2} \operatorname{sign}\left(S_{f}\right)
\end{aligned}
$$

The closed loop stability by the proposed control input with sliding surface together with the existence condition of the sliding mode will be investigated in next Theorem 1 .

Theorem 1: If the sliding surface (7) is designed in the stable, i.e. stable design of $K(x)$, the proposed input (21) with Assumption A1-A6 satisfies the existence condition of the sliding mode on the integral sliding surface and exponential stability.

Proof(Lee, 2010b); Take a Lyapunov function candidate as

$$
V(x)=\frac{1}{2} S_{f}^{T} S_{f}
$$

Differentiating (27) with respect to time leads to and substituting (26) into (28)

$$
\begin{aligned}
\dot{V}(x) & =S_{f}^{T} \dot{S}_{f} \\
& =S_{f}^{T} L_{1} \Delta f(x, t) x-S_{f}^{T} L_{1} \Delta g(x, t) K(x) x-S_{f}^{T}[I+\Delta I] L_{1} g_{0}(x, t) \Delta K x \\
& -S_{f}^{T}[I+\Delta I] L_{1} g_{0}(x, t) K_{1} S_{f}+S_{f}^{T} L_{1} \bar{d}(x, t)-S_{f}^{T}[I+\Delta I] L_{1} g_{0}(x, t) K_{2} \operatorname{sign}\left(S_{f}\right) \\
& \leq-\varepsilon K_{1}{ }^{\prime}|| S_{f}||^{2}, \quad \varepsilon=\min \{|| I+\Delta I||\} \\
& =-\varepsilon K_{1}{ }^{\prime} S_{f}^{T} S_{f} \\
& =-2 \varepsilon K_{1}{ }^{\prime} V(x)
\end{aligned}
$$

The second requirement to remove the reaching phase is satisfied. Therefore, the reaching phase is completely removed. There are no reaching phase problems. As a result, the real output dynamics can be exactly predetermined by the ideal sliding output with the matched uncertainty. From (28), the following equations are obtained as

$$
\dot{V}(x)+2 \varepsilon K_{1}^{\prime} V(x) \leq 0
$$




$$
V(x(t)) \leq V(x(0)) e^{-2 \varepsilon K_{1} t}
$$

And the second order derivative of $V(x)$ becomes

$$
\ddot{V}(x)=\dot{S}_{f}^{T} \dot{S}_{f}+S_{f}^{T} \ddot{S}_{f}=|| \dot{S}_{f}||^{2}+S_{f}\left(L_{1} C \ddot{x}+L_{0} \dot{x}\right)<\infty
$$

and by Assumption A5 $\ddot{V}(x)$ is bounded, which completes the proof of Theorem 1 .

\subsubsection{Continuous approximation of full sate feedback discontinuous control input}

The discontinuous control input (21) with (7) chatters from the beginning without reaching phase. The chattering of the discontinuous control input (21) may be harmful to the real dynamic plant. Hence using the saturation function for a suitable $\delta_{f}$, one make the input be continuous for practical application as

$$
u_{f c}=-K(x) x-K_{1} S_{f}-\left\{\Delta K x+K_{2} \operatorname{sign}\left(S_{f}\right)\right\} \frac{S_{f}}{\left|S_{f}\right|+\delta_{f}}
$$

which is different from that of (Chern \& Wu, 1992) continuous approximation. For a first order system, this approximation is the same as that of (Chern \& Wu, 1992) continuous approximation, but for a higher order system more than the first one, continuous approximation can be effectively made. The discontinuity of the control input can be dramatically improved without severe output performance deterioration.

\subsection{Practical output feedback integral variable structure controller}

For the implementation of the output feedback when full-state is not available, some additional assumptions are made

A7: The nominal input matrix $g_{0}(x, t)$ is constant, i.e, $g_{0}(x, t)=B$

A8: The unmatched $\Delta f(x, t)$, matched $\Delta g(x, t)$, and matched $d(x, t)$ are unknown and bounded and satisfied by the following conditions:

$$
\begin{gathered}
\Delta f(x, t)=\Delta f^{\prime}(x, t) C^{T} C=\Delta f^{\prime \prime}(x, t) C \\
\Delta g(x, t)=B B^{T} \Delta g^{\prime}(x, t)=B \Delta I, \quad 0 \leq|\Delta I| \leq p<1 \\
d(x, t)=B B^{T} d^{\prime}(x, t)=B d^{\prime \prime}(x, t)
\end{gathered}
$$

\subsubsection{Transformed output feedback integral sliding surface}

Now, the integral of the output is augmented as follows:

$$
\begin{aligned}
& \dot{y}_{0}(t)=A_{0} \cdot y(t), \quad y_{0}(0) \\
& y_{0}(t)=A_{0} \cdot \int_{0}^{t} y(\tau) d \tau+y_{0}(0)
\end{aligned}
$$

where $y_{0}(t) \in R^{r}, r \leq q$ is the integral of the output and $y_{0}(0)$ is the initial condition of the integral state determined later, and $A_{0}$ is appropriately dimensioned without loss of generality, $A_{0}=I$. 


\section{Assumption}

A9: $\left(H_{1} C B\right)$ has the inverse for some non zero row vector $H_{1}$.

Now, a transformed output feedback integral sliding surface is suggested be

$$
\begin{gathered}
S_{0}=\left(H_{1} C B\right)^{-1} \cdot\left(H_{1} \cdot y+H_{0} \cdot y_{0}\right)(=0) \\
y_{0}(0)=-H_{0}^{-} H_{1} \cdot y(0)
\end{gathered}
$$

where $H_{0}^{-}=\left(H_{0}^{T} W H_{0}\right)^{-1} H_{0}^{T} W$, which is transformed for easy proof of the existence condition of the sliding mode on the sliding surface as the works of (Decarlo et al., 1988) and (Lee, 2010b). In (35), non zero row vector $H_{0}$ and $H_{1}$ are the design parameters satisfying the following relationship

$$
H_{1} C\left[f_{0}(x, t)-B G(y) C\right]+H_{0} C=H_{1} C f_{0 c}(x, t)+H_{0} C=0
$$

where $f_{0 c}(x, t)=f_{0}(x, t)-B G(y) C$ is a closed loop system matrix and $G(y)$ is an output feedback gain. At $t=0$, this output feedback integral sliding surface is zero so that there will be no reaching phase(Lee \& Youn, 1994). In (35), $H_{0}$ and $H_{1}$ are the non zero row vectors as the design parameters such that the following assumption is satisfied.

\section{Assumption}

A10: $H_{1} \mathrm{Cg}(x, t)$ has the full rank and is invertible The equivalent control input is obtained using as

$$
u_{e q}=-\left[H_{1} C g(x, t)\right]^{-1}\left[H_{1} C f(x, t) x+H_{0} y_{0}(t)\right]-\left[H_{1} C g(x, t)\right]^{-1}\left[H_{1} C \Delta f(x, t)+\bar{d}(x, t)\right]
$$

This control input can not be implemented because of the uncertainties and disturbances. The ideal sliding mode dynamics of the output feedback integral sliding surface (35) can be derived by the equivalent control approach as (Decarlo et al., 1998)

$$
\begin{gathered}
\dot{x}_{s}=\left[f_{0}\left(x_{s}, t\right)-B\left(H_{1} C B\right)^{-1} H_{1} C f_{0}\left(x_{s}, t\right)-B\left(H_{1} C B\right)^{-1} H_{0} C\right] x_{s}, \quad x_{s}(0)=x(0) \\
y_{s}=C \cdot x_{s}
\end{gathered}
$$

and from $\dot{S}_{0}=0$, the another ideal sliding mode dynamics is obtained as(Lee, 2010a)

$$
\dot{y}_{s}=-H_{1}^{-} H_{0} y_{s}, \quad y_{s}(0)
$$

where $H_{1}^{-}=\left(H_{1}^{T} W H_{1}\right)^{-1} H_{1}^{T} W$. The solution of (39) or (41) identically defines the output feedback integral sliding surface. Hence to design the output feedback integral sliding surface as stable, this ideal sliding dynamics (39) is designed to be stable. To choose the stable gain based on the Lyapunov stability theory, the ideal sliding dynamics (39) is represented by the nominal plant of (3) as

$$
\begin{aligned}
\dot{x} & =f_{0}(x, t) x+g_{0}(x, t) u, \quad u=-G(y) y \\
& =f_{0 c}(x, t) x
\end{aligned}
$$

To select the stable gain, take a Lyapunov function candidate as 


$$
V(x)=x^{T} P x, \quad P>0
$$

The derivative of (43) becomes

$$
\dot{V}(x)=x^{T}\left[f_{0}(x, t)^{T} P+P f_{0}(x, t)\right] x+u^{T} g_{0}^{T}(x, t) P x+x^{T} P_{g_{0}}(x, t) u
$$

By means of the Lyapunov control theory(Khalil, 1996), take the control input as

$$
u=-g_{0}^{T}(x, t) P y=-B^{T} P y
$$

and $Q(x, t)>0$ and $Q_{c}(x, t)>0$ for all $x \in R^{n}$ and all $t \geq 0$ is

$$
\begin{aligned}
& f_{0}^{T}(x, t) P+P f_{0}(x, t)=-Q(x, t) \\
& f_{0 c}^{T}(x, t) P+P f_{0 c}(x, t)=-Q_{c}(x, t)
\end{aligned}
$$

then

$$
\begin{aligned}
\dot{V}(x) & =-x^{T} Q(x, t) x-x^{T} C^{T} P B B^{T} P x-x^{T} P B B^{T} P C x \\
& =-x^{T}\left[Q(x, t)+C^{T} P B B^{T} P+P B B^{T} P C\right] x \\
& =-x^{T}\left[f_{0 c}^{T}(x, t) P+P f_{0 c}(x, t)\right] x \\
& =-x^{T} Q_{c}(x, t) x \\
& \leq-\lambda_{\min }\left\{Q_{c}(x, t)\right\} x
\end{aligned}
$$

Therefore the stable gain is chosen as

$$
G(y)=B^{T} P \text { or }=\left(H_{1} C B\right)^{-1} H_{1} C f_{0}(x, t)
$$

\subsubsection{Output feedback discontinuous control input}

A corresponding output feedback discontinuous control input is proposed as follows:

$$
u_{0}=-G(y) y-\Delta G y-G_{1} S_{0}-G_{2} \operatorname{sign}\left(S_{0}\right)
$$

where $G(y)$ is a nonlinear output feedback gain satisfying the relationship (37) and (49), $\Delta G$ is a switching gain of the state, $G_{1}$ is a feedback gain of the output feedback integral sliding surface, and $G_{2}$ is a switching gain, respectively as

$$
\Delta g_{i}=\left\{\begin{array}{cc}
\Delta G=\left[\Delta g_{i}\right] \quad i=1, \ldots, q & \operatorname{sign}\left(S_{0} y_{i}\right)>0 \\
\leq \frac{\min \left\{\left(H_{1} C B\right)^{-1} H_{1} C \Delta f^{\prime \prime}(x . t)+\Delta I\left(H_{1} C B\right)^{-1} H_{1} C \Delta f^{\prime \prime}(x . t)+\Delta I\left(H_{1} C B\right)^{-1} H_{1} f_{0}(x, t)\right\}_{i}}{\min \{I+\Delta I\}} & \operatorname{sign}\left(S_{0} y_{i}\right)<0
\end{array}\right.
$$




$$
G_{2}=\frac{\max \left\{\left|d^{\prime \prime}(x, t)\right|\right\}}{\min \{I+\Delta I\}}
$$

The real sliding dynamics by the proposed control (50) with the output feedback integral sliding surface (35) is obtained as follows:

$$
\begin{aligned}
\dot{S}_{0}= & \left(H_{1} C B\right)^{-1}\left[H_{1} \dot{y}+H_{0} y\right] \\
= & \left(H_{1} C B\right)^{-1}\left[H_{1} C f_{0}(x, t) x+H_{1} C \Delta f(x, t)+H_{1} C(B+\Delta g(x, t)) u+H_{1} C \bar{d}(x, t)+H_{0} y\right] \\
= & \left(H_{1} C B\right)^{-1}\left[H_{1} C f_{0}(x, t) x-H_{1} C B G(y) y+H_{0} y\right] \\
& +\left(H_{1} C B\right)^{-1}\left[H_{1} C \Delta f(x, t)+H_{1} C \Delta g(x, t) K(y) y\right] \\
& +\left(H_{1} C B\right)^{-1}\left[H_{1} C(B+\Delta g(x, t))\left(-\Delta G y-G_{1} S_{0}-G_{2} \operatorname{sign}\left(S_{0}\right)+H_{1} C \bar{d}(x, t)\right]\right. \\
= & \left(H_{1} C B\right)^{-1}\left[H_{1} C \Delta f^{\prime \prime}(x, t) C x+H_{1} C \Delta g(x, t) G(y) y\right]-(I+\Delta I) \Delta G(y) y \\
& +\left[(I+\Delta I)\left(-G_{1} S_{0}-G_{2} \operatorname{sign}\left(S_{0}\right)\right)+d^{\prime \prime}(x, t)\right] \\
= & \left(H_{1} C B\right)^{-1} H_{1} C \Delta f^{\prime \prime}(x, t) y+\Delta I\left(H_{1} C B\right)^{-1} H_{1} f_{0}(x, t) y-(I+\Delta I) \Delta G(y) y \\
& +(I+\Delta I)\left(-G_{1} S_{0}-G_{2} \operatorname{sign}\left(S_{0}\right)\right)+d^{\prime \prime}(x, t)
\end{aligned}
$$

The closed loop stability by the proposed control input with the output feedback integral sliding surface together with the existence condition of the sliding mode will be investigated in next Theorem 1 .

Theorem 2: If the output feedback integral sliding surface (35) is designed to be stable, i.e. stable design of $G(y)$, the proposed control input (50) with Assumption A1-A10 satisfies the existence condition of the sliding mode on the output feedback integral sliding surface and closed loop exponential stability.

Proof; Take a Lyapunov function candidate as

$$
V(y)=\frac{1}{2} S_{0}^{T} S_{0}
$$

Differentiating (57) with respect to time leads to and substituting (56) into (58)

$$
\begin{aligned}
\dot{V}(y)= & S_{0}^{T} \dot{S}_{0} \\
= & S_{0}^{T}\left[\left(H_{1} C B\right)^{-1} H_{1} C \Delta f^{\prime \prime}(x, t)+\Delta I\left(H_{1} C B\right)^{-1} H_{1} f_{0}(x, t)\right] y-S_{0}^{T}(I+\Delta I) \Delta G(y) y \\
& +S_{0}^{T}(I+\Delta I)\left(-G_{1} S_{0}-G_{2} \operatorname{sign}\left(S_{0}\right)\right)+S_{0}^{T} d^{\prime \prime}(x, t) \\
\leq & -\varepsilon G_{1}|| S_{0}||^{2}, \quad \varepsilon=\min \{|| I+\Delta I||\} \\
= & -\varepsilon G_{1} S_{0}^{T} S_{0} \\
= & -2 \varepsilon G_{1} V(y)
\end{aligned}
$$

From (58), the second requirement to get rid of the reaching phase is satisfied. Therefore, the reaching phase is clearly removed. There are no reaching phase problems. As a result, the real output dynamics can be exactly predetermined by the ideal sliding output with the matched uncertainty. Moreover from (58), the following equations are obtained as

$$
\dot{V}(y)+2 \varepsilon G_{1} V(y) \leq 0
$$




$$
V(y(t)) \leq V(y(0)) e^{-2 \varepsilon G_{1} t}
$$

And the second order derivative of $V(x)$ becomes

$$
\ddot{V}(y)=\dot{S}_{0}^{T} \dot{S}_{0}+S_{0}^{T} \ddot{S}_{0}=|| \dot{S}_{0}||^{2}+S_{0}\left(H_{1} C B\right)^{-1}\left(H_{1} C \ddot{x}+H_{0} C \dot{x}\right)<\infty
$$

and by Assumption A5 $\ddot{V}(x)$ is bounded, which completes the proof of Theorem 2 .

\subsubsection{Continuous approximation of output feedback discontinuous control input}

Also, the control input (50) with (35) chatters from the beginning without reaching phase. The chattering of the discontinuous control input may be harmful to the real dynamic plant so it must be removed. Hence using the saturation function for a suitable $\delta_{0}$, one make the part of the discontinuous input be continuous effectively for practical application as

$$
u_{0 c}=-G(y) y-G_{1} S_{0}-\left\{\Delta G y+G_{2} \operatorname{sign}\left(S_{0}\right)\right\} \frac{S_{0}}{\left|S_{0}\right|+\delta_{0}}
$$

The discontinuity of control input of can be dramatically improved without severe output performance deterioration.

\section{Design examples and simulation studies}

\subsection{Example 1: Full-state feedback practical integral variable structure controller}

Consider a second order affine uncertain nonlinear system with mismatched uncertainties and matched disturbance

$$
\begin{gathered}
\dot{x}_{1}=-x_{1}+0.1 x_{1} \sin ^{2}\left(x_{1}\right)+x_{2}+0.02 \sin \left(2.0 x_{1}\right) u \\
\dot{x}_{2}=x_{2}+x_{2} \sin ^{2}\left(x_{2}\right)+(2.0+0.5 \sin (2.0 t)) u+\bar{d}(x, t) \\
\bar{d}(x, t)=0.7 \sin \left(x_{1}\right)-0.8 \sin \left(x_{2}\right)+0.2\left(x_{1}^{2}+x_{2}^{2}\right)+2.0 \sin (5.0 t)+3.0
\end{gathered}
$$

Since (63) satisfy the Assumption A1, (63) is represented in state dependent coefficient form as

$$
\left[\begin{array}{l}
\dot{x}_{1} \\
\dot{x}_{2}
\end{array}\right]=\left[\begin{array}{cc}
-1+0.1 \sin ^{2}\left(x_{1}\right) & 1 \\
0 & 1+\sin ^{2}\left(x_{2}\right)
\end{array}\right] \cdot\left[\begin{array}{l}
x_{1} \\
x_{2}
\end{array}\right]+\left[\begin{array}{c}
0.02 \sin \left(x_{1}\right) \\
2.0+0.5 \sin (2.0 t)
\end{array}\right] u+\left[\begin{array}{c}
0 \\
\bar{d}(x, t)
\end{array}\right]
$$

where the nominal parameter $f_{0}(x, t)$ and $g_{0}(x, t)$ and mismatched uncertainties $\Delta f(x, t)$ and $\Delta g(x, t)$ are

$$
\begin{aligned}
& f_{0}(x, t)=\left[\begin{array}{cc}
-1 & 1 \\
0 & 1
\end{array}\right], g_{0}(x, t)=\left[\begin{array}{c}
0 \\
2.0
\end{array}\right], \Delta f(x, t)=\left[\begin{array}{cc}
0.1 \sin ^{2}\left(x_{1}\right) & 0 \\
0 & \sin ^{2}\left(x_{2}\right)
\end{array}\right] \\
& \Delta g(x, t)=\left[\begin{array}{l}
0.02 \sin \left(x_{1}\right) \\
0.2 \sin (2.0 t)
\end{array}\right]
\end{aligned}
$$


To design the full-state feedback integral sliding surface, $f_{c}(x, t)$ is selected as

$$
f_{c}(x, t)=f_{0}(x, t)-g_{0}(x, t) K(x)=\left[\begin{array}{cc}
-1 & 1 \\
-70 & -21
\end{array}\right]
$$

in order to assign the two poles at -16.4772 and -5.5228 . Hence, the feedback gain $K(x)$ becomes

$$
K(x)=\left[\begin{array}{ll}
35 & 11
\end{array}\right]
$$

The $P$ in $(14)$ is chosen as

$$
P=\left[\begin{array}{cc}
100 & 17.5 \\
17.5 & 5.5
\end{array}\right]>0
$$

so as to be

$$
f_{c}^{T}(x, t) P+P f_{c}(x, t)=\left[\begin{array}{cc}
-2650 & -670 \\
-670 & -196
\end{array}\right]<0
$$

Hence, the continuous static feedback gain is chosen as

$$
K(x)=g_{0}^{T}(x, t) P=\left[\begin{array}{ll}
35 & 11
\end{array}\right]
$$

Therefore, the coefficient of the sliding surface is determined as

$$
L_{1}=\left[\begin{array}{ll}
L_{11} & L_{12}
\end{array}\right]=\left[\begin{array}{ll}
10 & 1
\end{array}\right]
$$

Then, to satisfy the relationship (8a) and from (8b), $L_{0}$ is selected as

$$
L_{0}=-L_{1}\left[f_{0}(x, t)-g_{0}(x, t) K(x)\right]=-L_{1} f_{c}(x, t)=\left[L_{11}+70 L_{12} \quad-L_{11}+21 L_{12}\right]=\left[\begin{array}{ll}
80 & 11
\end{array}\right]
$$

The selected gains in the control input (21), (23)-(25) are as follows:

$$
\begin{gathered}
\Delta k_{1}=\left\{\begin{array}{lll}
+4.0 & \text { if } \quad S_{f} x_{1}>0 \\
-4.0 & \text { if } \quad S_{f} x_{1}<0
\end{array}\right. \\
\Delta k_{2}= \begin{cases}+5.0 & \text { if } \quad S_{f} x_{2}>0 \\
-5.0 & \text { if } \quad S_{f} x_{2}<0\end{cases} \\
K_{1}=400.0 \\
K_{2}=2.8+0.2\left(x_{1}^{2}+x_{2}^{2}\right)
\end{gathered}
$$

The simulation is carried out under $1[\mathrm{msec}]$ sampling time and with $x(0)=\left[\begin{array}{ll}10 & 5\end{array}\right]^{T}$ initial state. Fig. 1 shows four case $x_{1}$ and $x_{2}$ time trajectories (i)ideal sliding output, (ii) no uncertainty and no disturbance (iii)matched uncertainty/disturbance, and (iv)unmatched 
uncertainty and matched disturbance. The three case output responses except the case (iv) are almost identical to each other. The four phase trajectories (i)ideal sliding trajectory, (ii)no uncertainty and no disturbance (iii)matched uncertainty/disturbance, and (iv) unmatched uncertainty and matched disturbance are depicted in Fig. 2. As can be seen, the sliding surface is exactly defined from a given initial condition to the origin, so there is no reaching phase, only the sliding exists from the initial condition. The one of the two main problems of the VSS is removed and solved. The unmatched uncertainties influence on the ideal sliding dynamics as in the case (iv). The sliding surface $S_{f}(t)$ (i) unmatched uncertainty and matched disturbance is shown in Fig. 3. The control input (i) unmatched uncertainty and matched disturbance is depicted in Fig. 4. For practical application, the discontinuous input is made be continuous by the saturation function with a new form as in (32) for a positive $\delta_{f}=0.8$. The output responses of the continuous input by (32) are shown in Fig. 5 for the four cases (i)ideal sliding output, (ii)no uncertainty and no disturbance (iii)matched uncertainty/disturbance, and (iv)unmatched uncertainty and matched disturbance. There is no chattering in output states. The four case trajectories (i)ideal sliding time trajectory, (ii)no uncertainty and no disturbance (iii)matched uncertainty/disturbance, and (iv) unmatched uncertainty and matched disturbance are depicted in Fig. 6. As can be seen, the trajectories are continuous. The four case sliding surfaces are shown in fig. 7 , those are continuous. The three case continuously implemented control inputs instead of the discontinuous input in Fig. 4 are shown in Fig. 8 without the severe performance degrade, which means that the continuous VSS algorithm is practically applicable. The another of the two main problems of the VSS is improved effectively and removed.

From the simulation studies, the usefulness of the proposed SMC is proven.

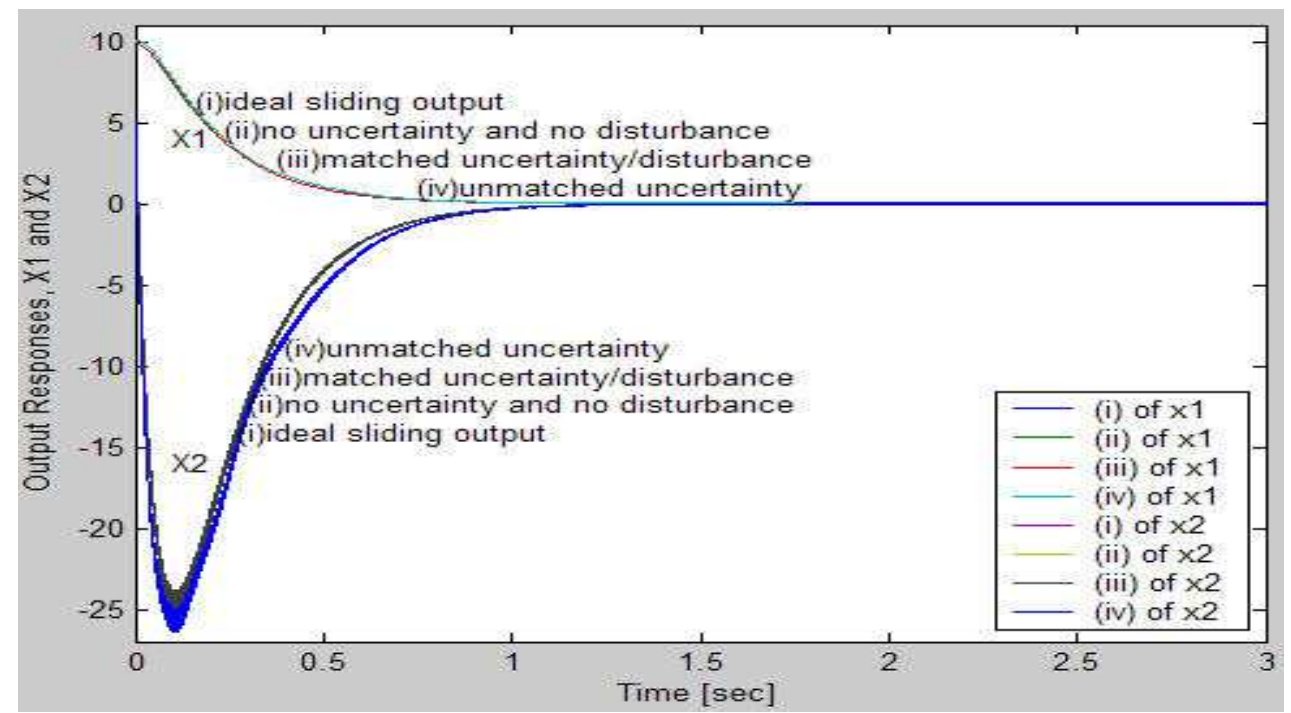

Fig. 1. Four case $x_{1}$ and $x_{2}$ time trajectories (i)ideal sliding output, (ii) no uncertainty and no disturbance (iii)matched uncertainty/disturbance, and (iv)unmatched uncertainty and matched disturbance 


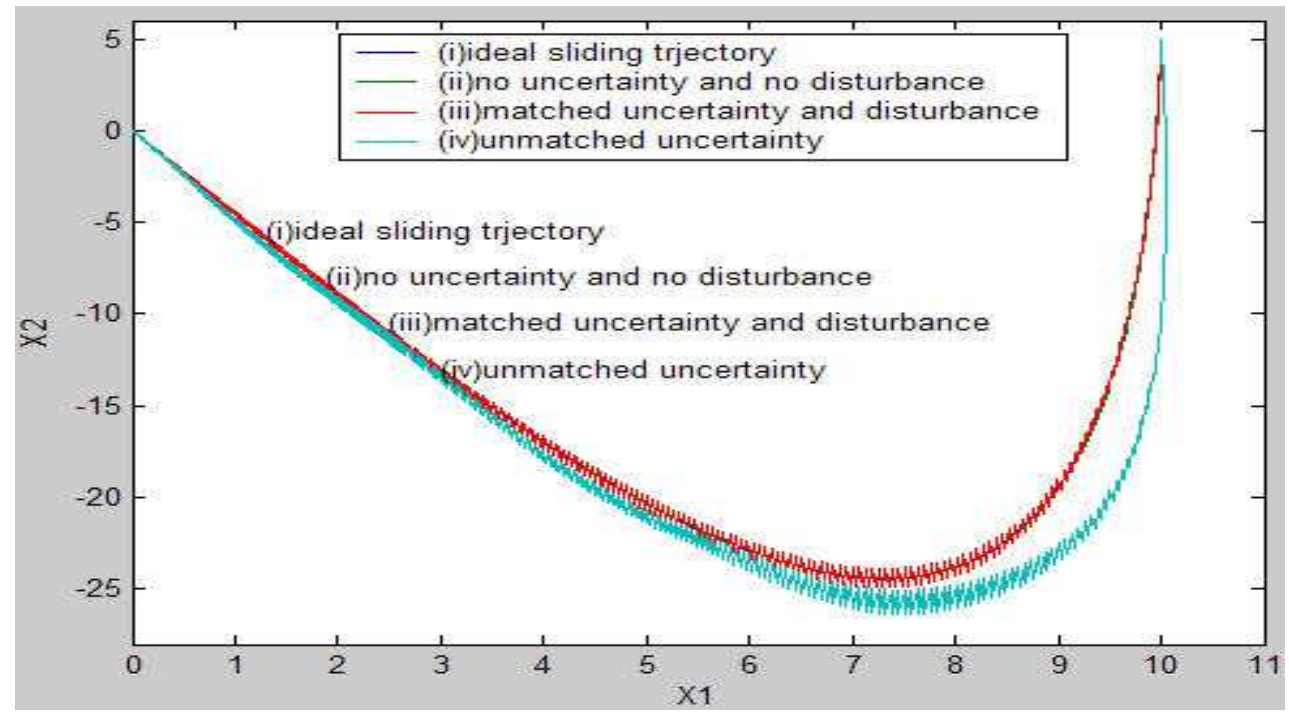

Fig. 2. Four phase trajectories (i)ideal sliding trajectory, (ii)no uncertainty and no disturbance (iii)matched uncertainty/disturbance, and (iv) unmatched uncertainty and matched disturbance

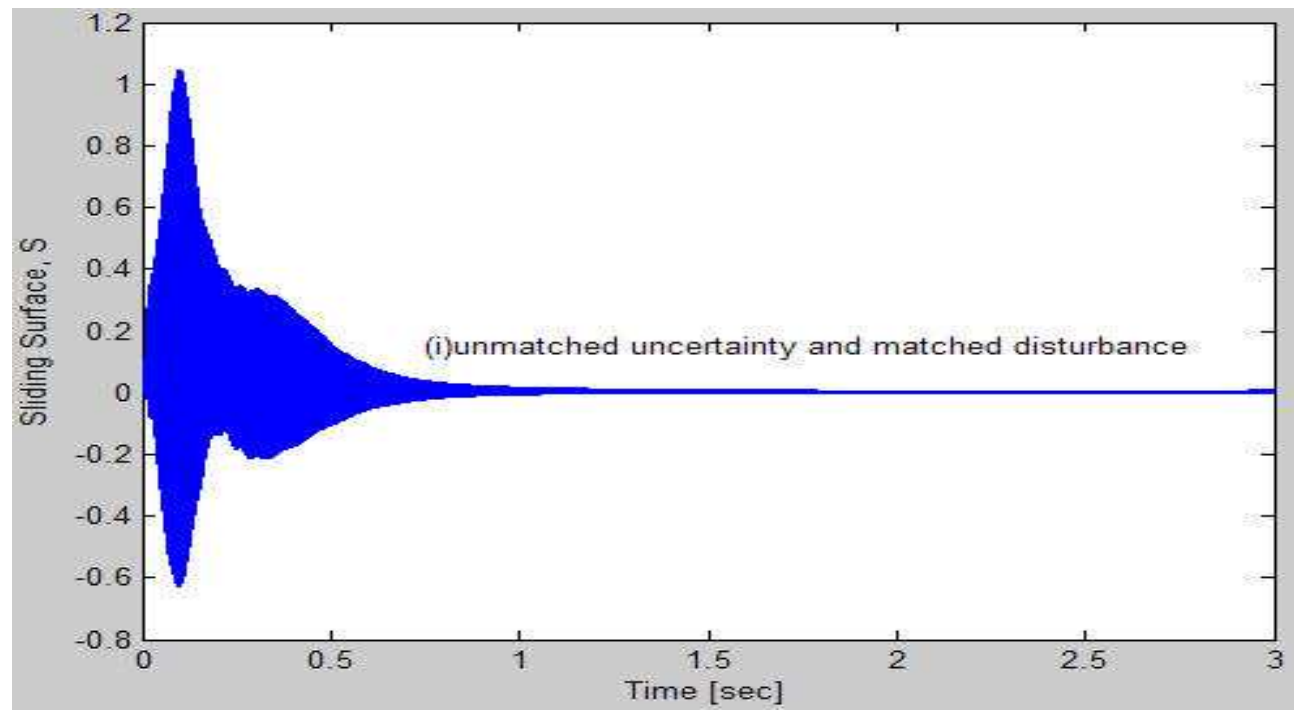

Fig. 3. Sliding surface $S_{f}(t)$ (i) unmatched uncertainty and matched disturbance 


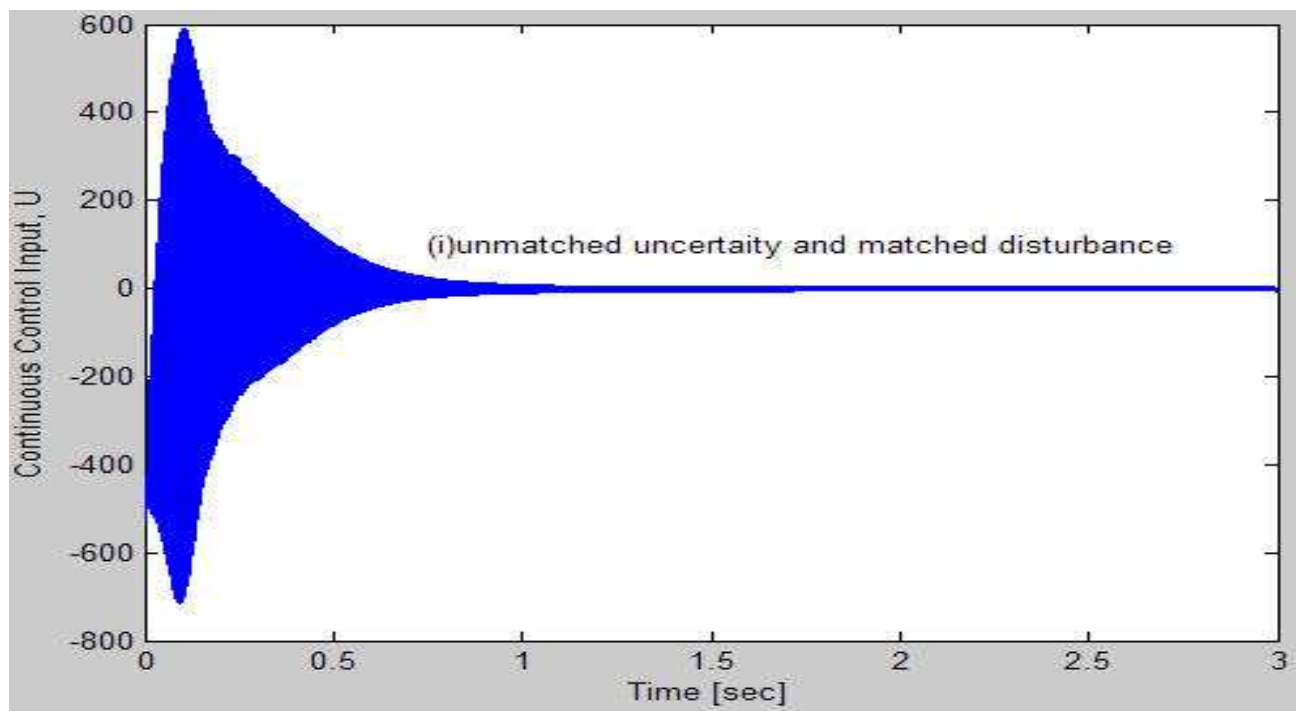

Fig. 4. Discontinuous control input (i) unmatched uncertainty and matched disturbance

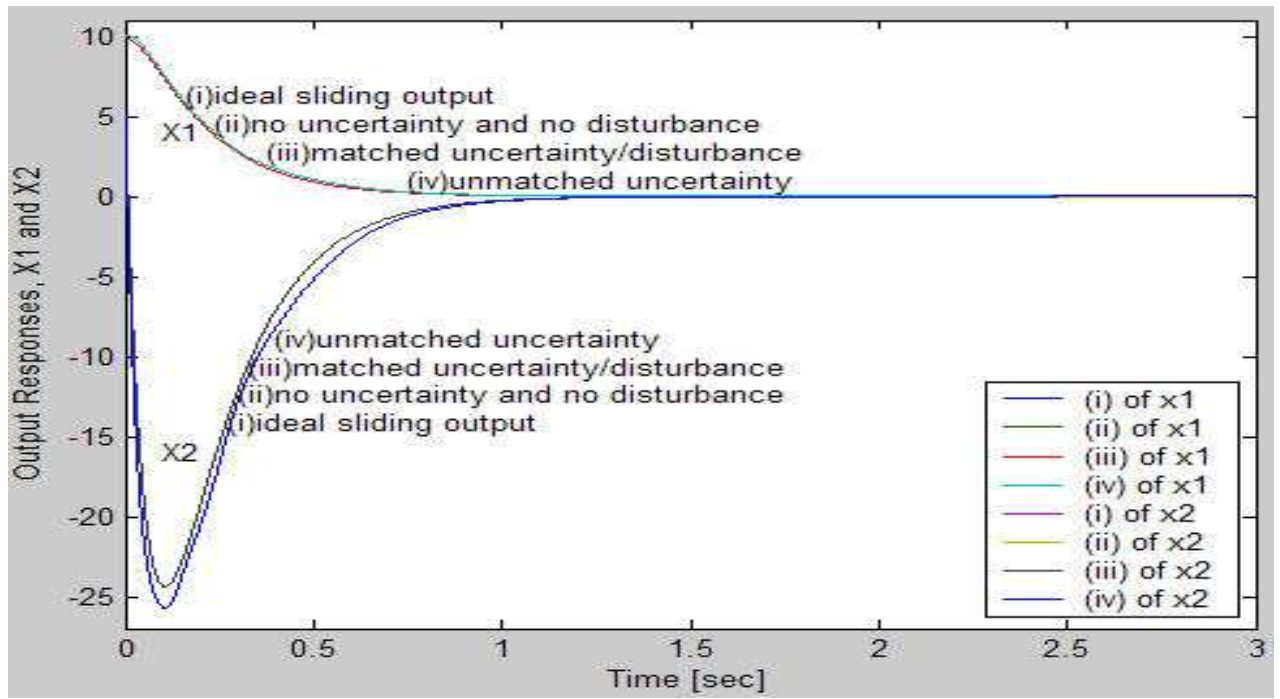

Fig. 5. Four case $x_{1}$ and $x_{2}$ time trajectories (i)ideal sliding output, (ii) no uncertainty and no disturbance (iii)matched uncertainty/disturbance, and (iv)unmatched uncertainty and matched disturbance by the continuously approximated input for a positive $\delta_{f}=0.8$ 


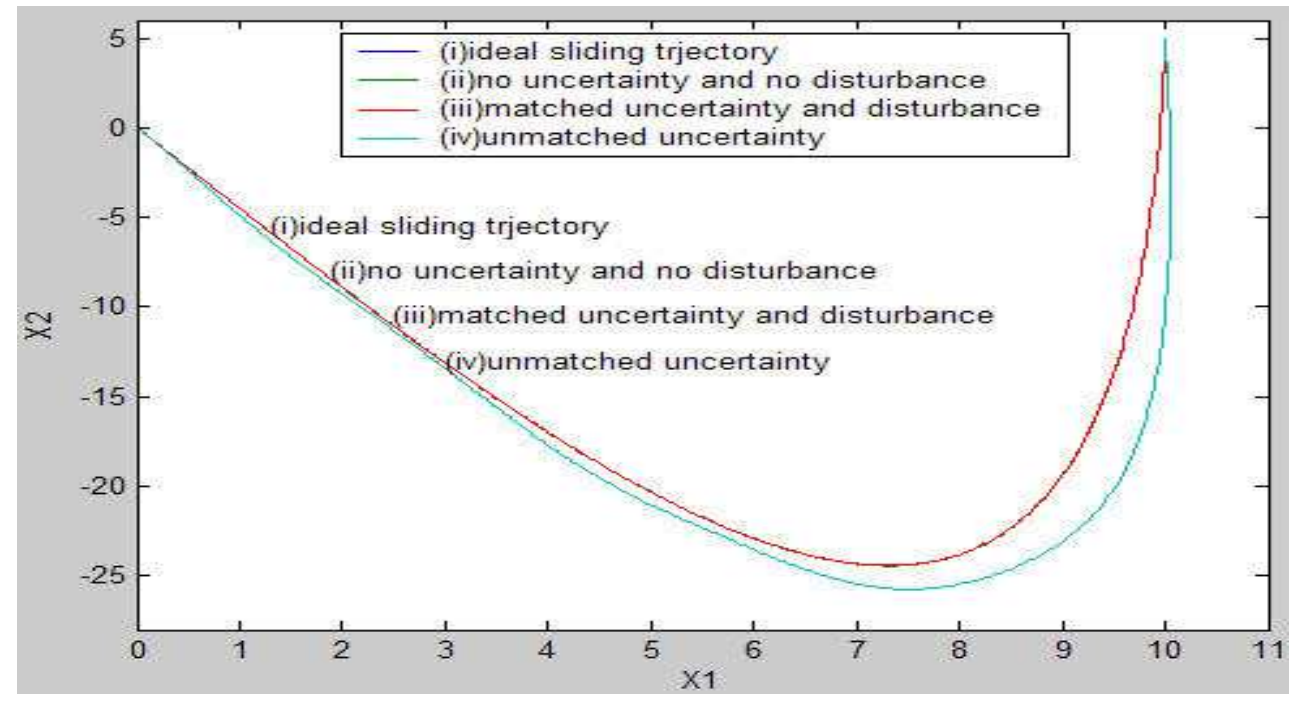

Fig. 6. Four phase trajectories (i)ideal sliding trajectory, (ii)no uncertainty and no disturbance (iii)matched uncertainty/disturbance, and (iv) unmatched uncertainty and matched disturbance by the continuously approximated input

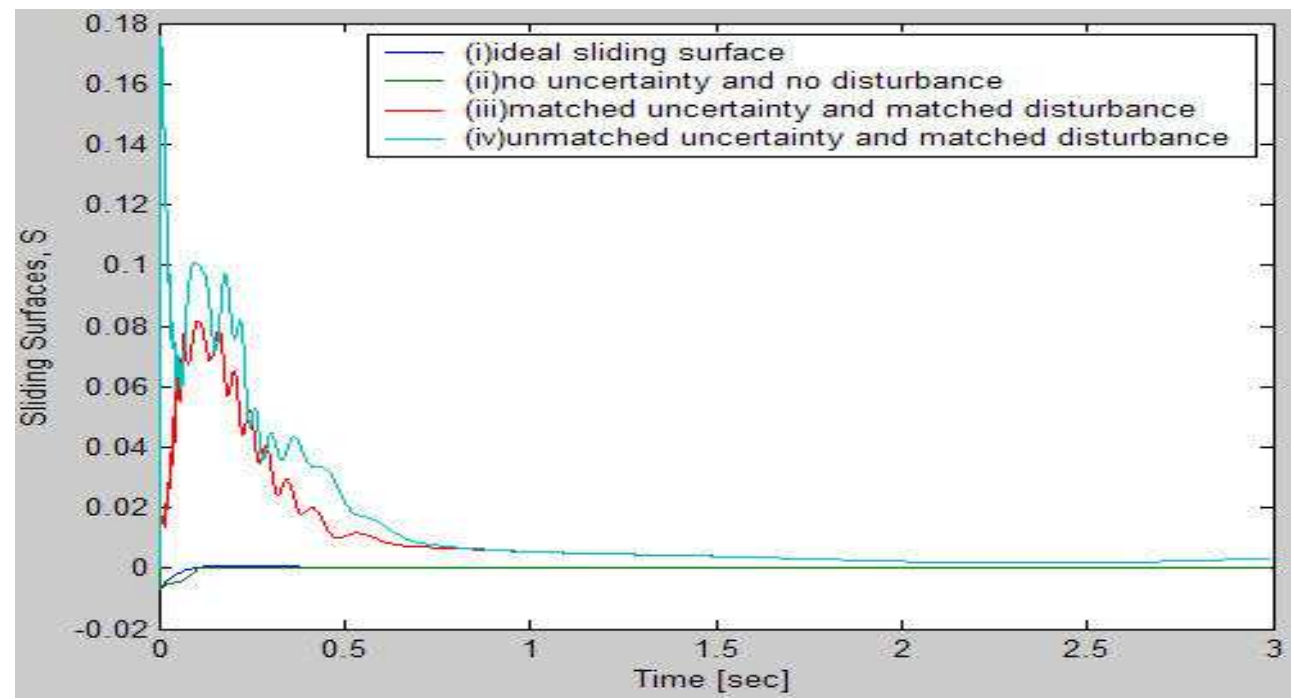

Fig. 7. Four sliding surfaces (i)ideal sliding surface, (ii)no uncertainty and no disturbance (iii)matched uncertainty/disturbance, and (iv) unmatched uncertainty and matched disturbance by the continuously approximated input 


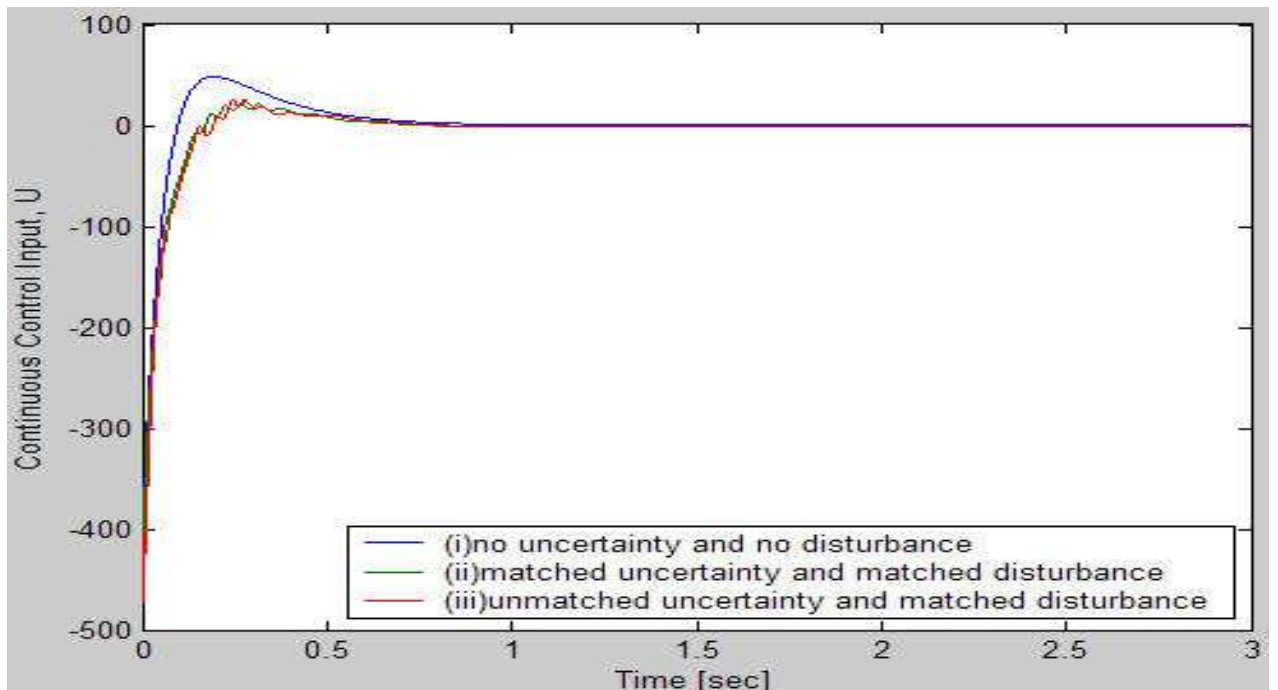

Fig. 8. Three case continuous control inputs $u_{f c}$ (i)no uncertainty and no disturbance (ii)matched uncertainty/disturbance, and (iii) unmatched uncertainty and matched

\subsection{Example 2: Output feedback practical integral variable structure controller}

Consider a third order uncertain affine nonlinear system with unmatched system matrix uncertainties and matched input matrix uncertainties and disturbance

$$
\begin{gathered}
{\left[\begin{array}{l}
\dot{x}_{1} \\
\dot{x}_{2} \\
\dot{x}_{3}
\end{array}\right]=\left[\begin{array}{ccc}
-3-3 \sin ^{2}\left(x_{1}\right) & 1 & 0 \\
0 & -1 & 1 \\
1+0.5 \sin ^{2}\left(x_{2}\right) & 0 & 2+0.4 \sin ^{2}\left(x_{3}\right)
\end{array}\right]\left[\begin{array}{l}
x_{1} \\
x_{2} \\
x_{3}
\end{array}\right]+\left[\begin{array}{c}
0 \\
0 \\
2+0.3 \sin (2 t)
\end{array}\right] u+\left[\begin{array}{c}
0 \\
0 \\
\bar{d}_{1}(x, t)
\end{array}\right]} \\
y=\left[\begin{array}{lll}
1 & 0 & 0 \\
0 & 0 & 1
\end{array}\right]\left[\begin{array}{l}
x_{1} \\
x_{2} \\
x_{3}
\end{array}\right] \\
\bar{d}_{1}(x, t)=0.7 \sin \left(x_{1}\right)-0.8 \sin \left(x_{2}\right)+0.2\left(x_{1}^{2}+x_{3}^{2}\right)+1.5 \sin (2 t)+1.5
\end{gathered}
$$

where the nominal matrices $f_{0}(x, t), g_{0}(x, t)=B$ and $C$, the unmatched system matrix uncertainties and matched input matrix uncertainties and matched disturbance are

$$
f_{0}(x, t)=\left[\begin{array}{ccc}
-3 & 1 & 0 \\
0 & -1 & 1 \\
1 & 0 & 2
\end{array}\right], B=\left[\begin{array}{l}
0 \\
0 \\
2
\end{array}\right], \quad C=\left[\begin{array}{lll}
1 & 0 & 0 \\
0 & 0 & 1
\end{array}\right], \Delta f=\left[\begin{array}{ccc}
-3 \sin ^{2}\left(x_{1}\right) & 0 & 0 \\
0 & 0 & 0 \\
0.5 \sin ^{2}\left(x_{2}\right) & 0 & 0.4 \sin ^{2}\left(x_{3}\right)
\end{array}\right]
$$




$$
\Delta g(x, t)=\left[\begin{array}{c}
0 \\
0 \\
0.3 \sin (2 t)
\end{array}\right], \quad \bar{d}(x, t)=\left[\begin{array}{c}
0 \\
0 \\
\bar{d}_{1}(x, t)
\end{array}\right]
$$

The eigenvalues of the open loop system matrix $f_{0}(x, t)$ are $-2.6920,-2.3569$, and 2.0489, hence $f_{0}(x, t)$ is unstable. The unmatched system matrix uncertainties and matched input matrix uncertainties and matched disturbance satisfy the assumption A3 and A8 as

$$
\Delta f^{\prime \prime}=\left[\begin{array}{cc}
-3 \sin ^{2}\left(x_{1}\right) & 0 \\
0 & 0 \\
0.5 \sin ^{2}\left(x_{2}\right) & 0.4 \sin ^{2}\left(x_{3}\right)
\end{array}\right], \quad \Delta I=0.15 \sin (2 t) \leq 0.15<1, \quad d^{\prime \prime}(x, t)=\frac{1}{2} \bar{d}_{1}(x, t)
$$

disturbance by the continuously approximated input for a positive $\delta_{f}=0.8$

To design the output feedback integral sliding surface, $f_{c}(x, t)$ is designed as

$$
f_{0 c}(x, t)=f_{0}(x, t)-B G(y) C=\left[\begin{array}{ccc}
-3 & 1 & 0 \\
0 & -1 & 1 \\
-19 & 0 & -30
\end{array}\right]
$$

in order to assign the three stable pole to $f_{c}(x, t)$ at -30.0251 and $-2.4875 \pm i 0.6636$. The constant feedback gain is designed as

$$
\begin{gathered}
G(y) C=2^{-1}\left\{\left[\begin{array}{lll}
1 & 0 & 2
\end{array}\right]-\left[\begin{array}{lll}
-19 & 0 & 30
\end{array}\right]\right\} \\
\therefore G(y)=\left[\begin{array}{ll}
10 & 16
\end{array}\right]
\end{gathered}
$$

Then, one find $H_{1}=\left[\begin{array}{ll}h_{11} & h_{12}\end{array}\right]$ and $H_{0}=\left[\begin{array}{ll}h_{01} & h_{02}\end{array}\right]$ which satisfy the relationship (37) as

$$
h_{11}=0, \quad h_{01}=19 h_{12}, \quad h_{02}=30 h_{12}
$$

One select $h_{12}=1, h_{01}=19$, and $h_{02}=30$. Hence $H_{1} C B=2 h_{12}=2$ is a non zero satisfying A4. The resultant output feedback integral sliding surface becomes

$$
S_{0}=\frac{1}{2}\left\{\left[\begin{array}{ll}
0 & 1
\end{array}\right]\left[\begin{array}{l}
y_{1} \\
y_{2}
\end{array}\right]+\left[\begin{array}{ll}
19 & 30
\end{array}\right]\left[\begin{array}{l}
y_{01} \\
y_{02}
\end{array}\right]\right\}
$$

where

$$
\begin{gathered}
y_{01}=\int_{0}^{t} y_{1}(\tau) d \tau \\
y_{02}=\int_{0}^{t} y_{2}(\tau) d \tau-y_{2}(0) / 30
\end{gathered}
$$

The output feedback control gains in (50), (51)-(55) are selected as follows: 


$$
\begin{gathered}
\Delta g_{1}=\left\{\begin{array}{lll}
+1.6 & \text { if } \quad S_{0} y_{1}>0 \\
-1.6 & \text { if } \quad S_{0} y_{1}<0
\end{array}\right. \\
\Delta g_{2}=\left\{\begin{array}{lll}
+1.7 & \text { if } S_{0} y_{2}>0 \\
-1.7 & \text { if } \quad S_{0} y_{2}<0
\end{array}\right. \\
G_{1}=500.0 \\
G_{2}=3.2+0.2\left(y_{1}^{2}+y_{2}^{2}\right)
\end{gathered}
$$

The simulation is carried out under $1[\mathrm{msec}]$ sampling time and with $x(0)=\left[\begin{array}{lll}10 & 0.0 & 5\end{array}\right]^{T}$ initial state. Fig. 9 shows the four case two output responses of $y_{1}$ and $y_{2}$ (i)ideal sliding output, (ii) with no uncertainty and no disturbance, (iii)with matched uncertainty and matched disturbance, and (iv) with ummatched uncertainty and matched disturbance. The each two output is insensitive to the matched uncertainty and matched disturbance, hence is almost equal, so that the output can be predicted. The four case phase trajectories (i)ideal sliding trajectory, (ii) with no uncertainty and no disturbance, (iii)with matched uncertainty and matched disturbance, and (iv) with ummatched uncertainty and matched disturbance are shown in Fig. 10. There is no reaching phase and each phase trajectory except the case (iv) with ummatched uncertainty and matched disturbance is almost identical also. The sliding surface is exactly defined from a given initial condition to the origin. The output feedback integral sliding surfaces (i) with ummatched uncertainty and matched disturbance is depicted in Fig. 11. Fig. 12 shows the control inputs (i)with unmatched uncertainty and matched disturbance. For practical implementation, the discontinuous input can be made continuous by the saturation function with a new form as in (32) for a positive $\delta_{0}=0.02$. The output responses by the continuous input of (62) are shown in Fig. 13 for the four cases (i)ideal sliding output, (ii)no uncertainty and no disturbance (iii)matched uncertainty/disturbance, and (iv)unmatched uncertainty and matched disturbance. There is no chattering in output responses. The four case trajectories (i)ideal sliding time trajectory, (ii)no uncertainty and no disturbance (iii)matched uncertainty/disturbance, and (iv) unmatched uncertainty and matched disturbance are depicted in Fig. 14. As can be seen, the trajectories are continuous. The four case sliding surfaces are shown in fig. 15, those are continuous also. The three case continuously implemented control inputs instead of the discontinuous input in Fig. 12 are shown in Fig. 16 without the severe performance loss, which means that the chattering of the control input is removed and the continuous VSS algorithm is practically applicable to the real dynamic plants. From the above simulation studies, the proposed algorithm has superior performance in view of the no reaching phase, complete robustness, predetermined output dynamics, the prediction of the output, and practical application. The effectiveness of the proposed output feedback integral nonlinear $\mathrm{SMC}$ is proven.

Through design examples and simulation studies, the usefulness of the proposed practical integral nonlinear variable structure controllers is verified. The continuous approximation VSS controllers without the reaching phase in this chapter can be practically applicable to the real dynamic plants. 


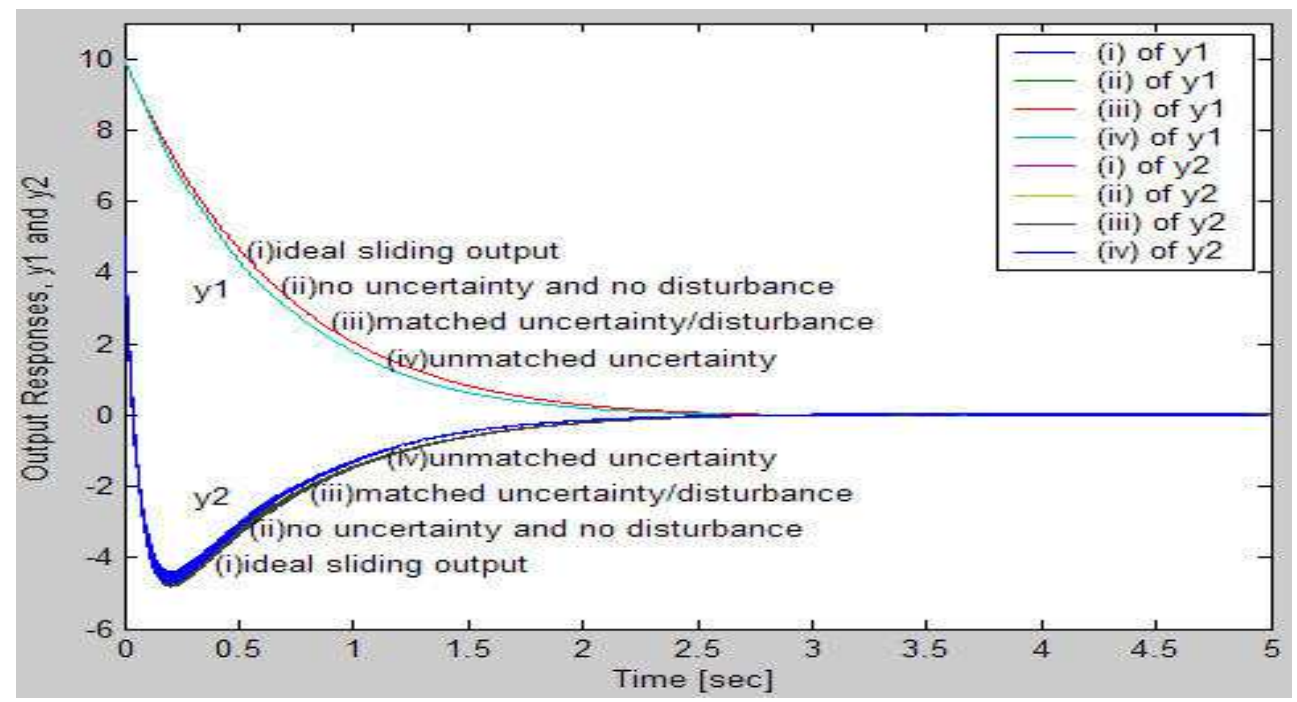

Fig. 9. Four case two output responses of $y_{1}$ and $y_{2}$ (i)ideal sliding output, (ii) with no uncertainty and no disturbance, (iii)with matched uncertainty and matched disturbance, and (iv) with ummatched uncertainty and matched disturbance

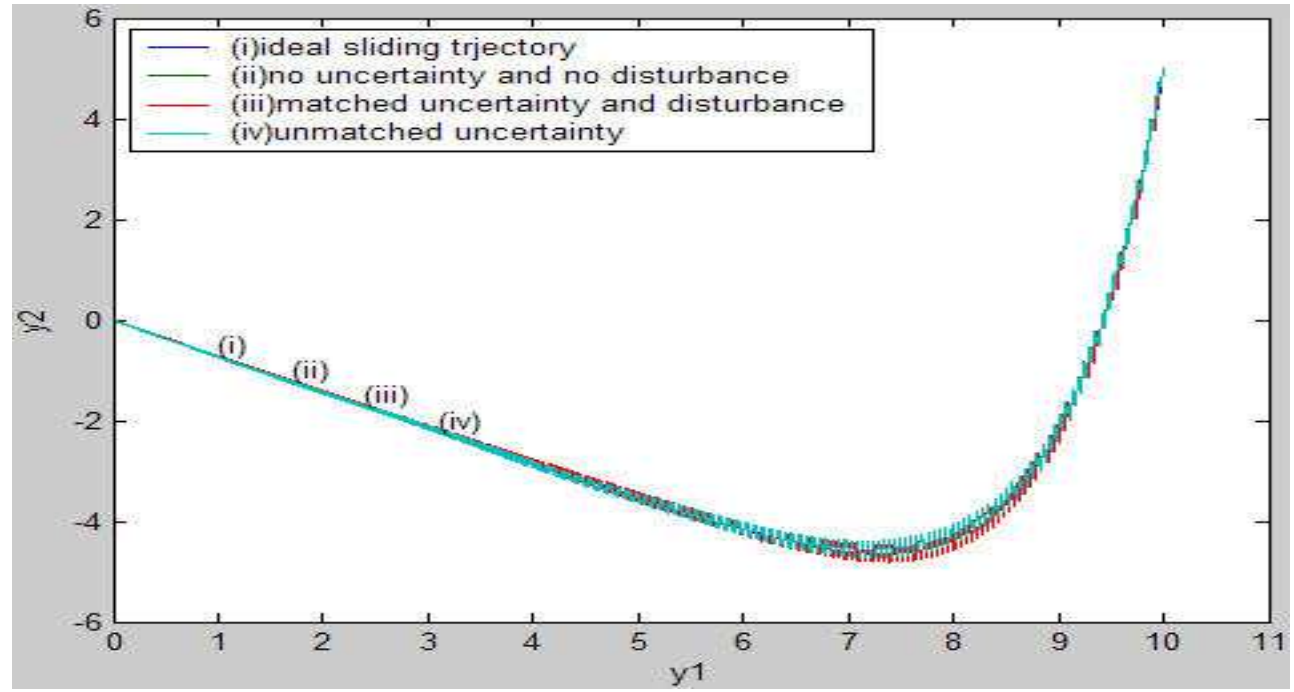

Fig. 10. Four phase trajectories (i)ideal sliding trajectory, (ii)no uncertainty and no disturbance (iii)matched uncertainty/disturbance, and (iv)unmatched uncertainty and matched disturbance 


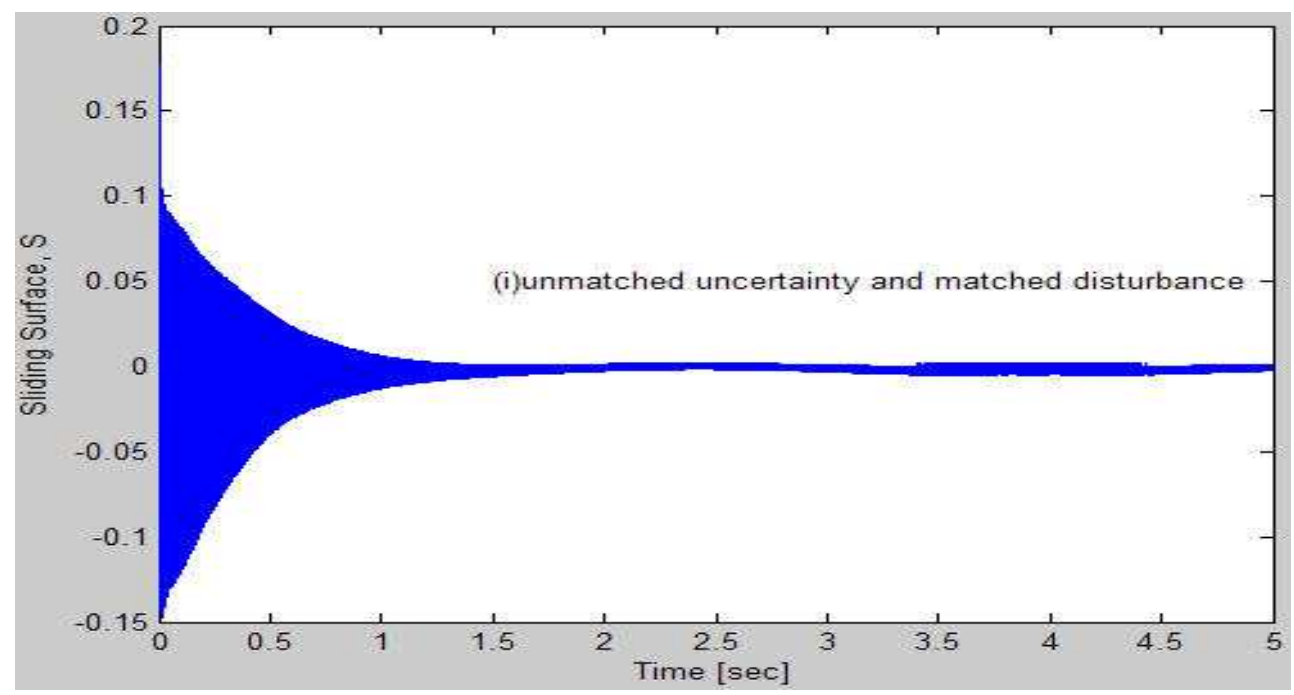

Fig. 11. Sliding surface $S_{0}(t)$ (i) unmatched uncertainty and matched disturbance

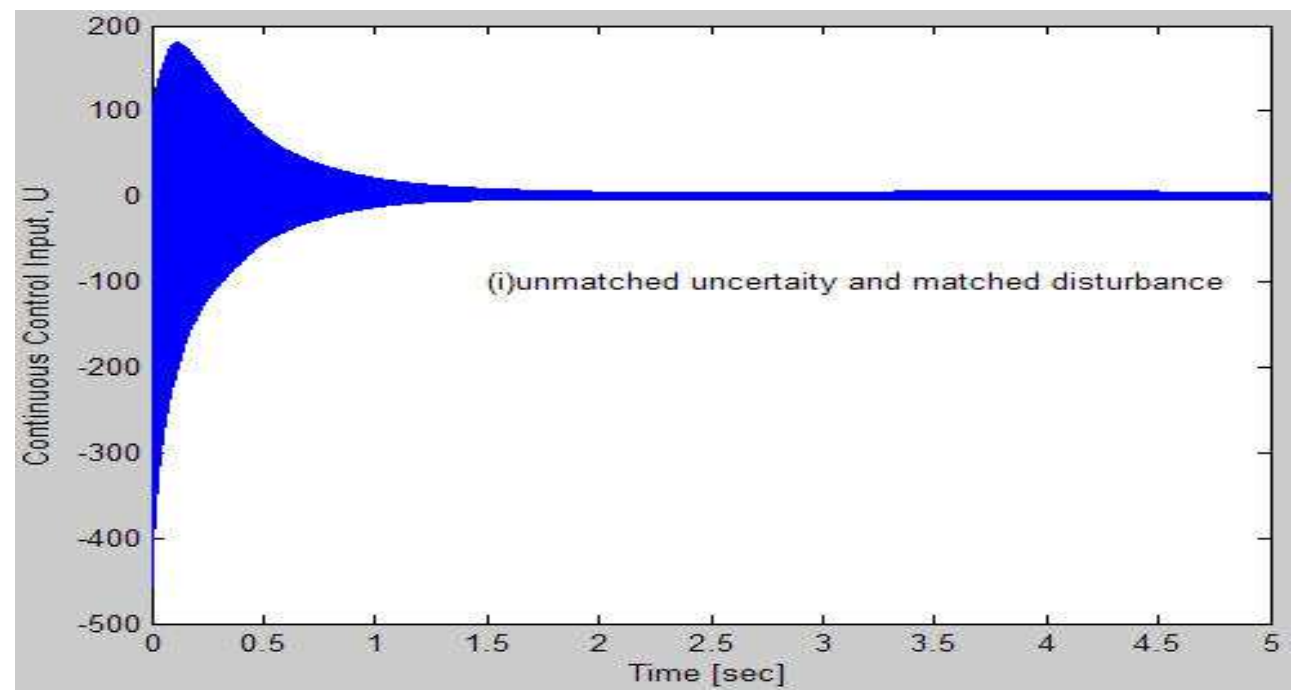

Fig. 12. Discontinuous control input (i) unmatched uncertainty and matched disturbance 


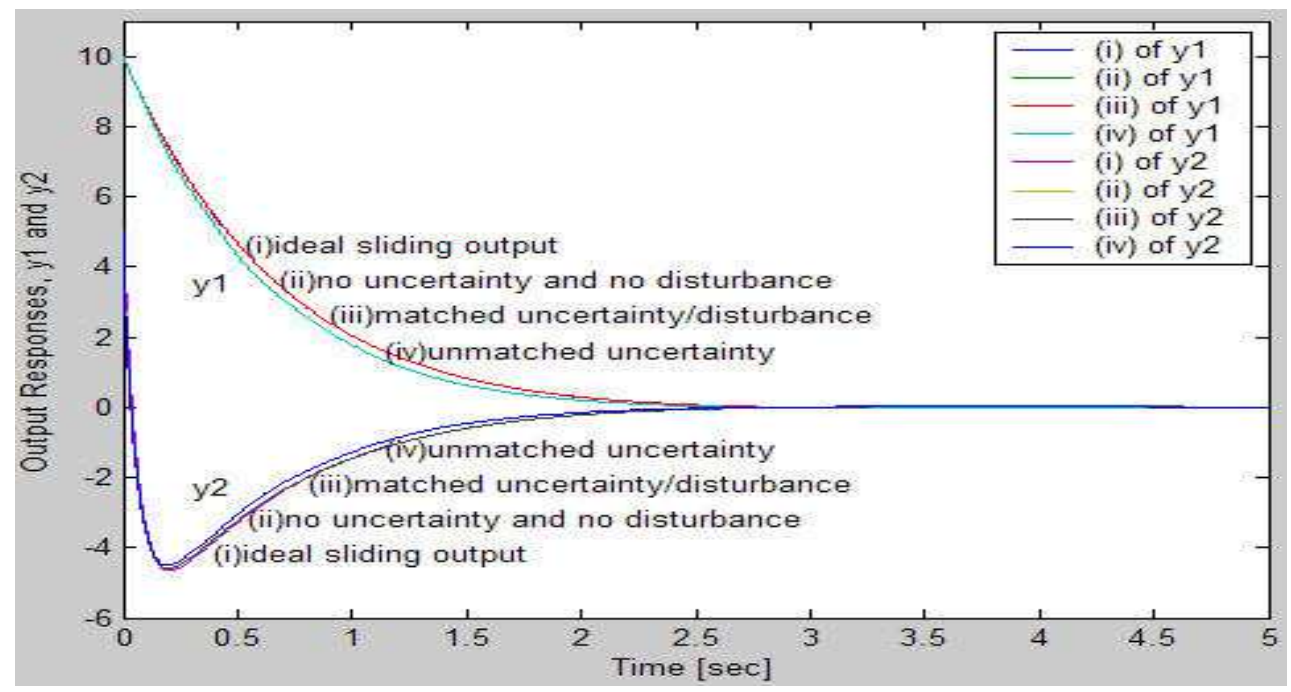

Fig. 13. Four case $y_{1}$ and $y_{2}$ time trajectories (i)ideal sliding output, (ii) no uncertainty and no disturbance (iii)matched uncertainty/disturbance, and (iv)unmatched uncertainty and matched disturbance by the continuously approximated input for a positive $\delta_{0}=0.02$

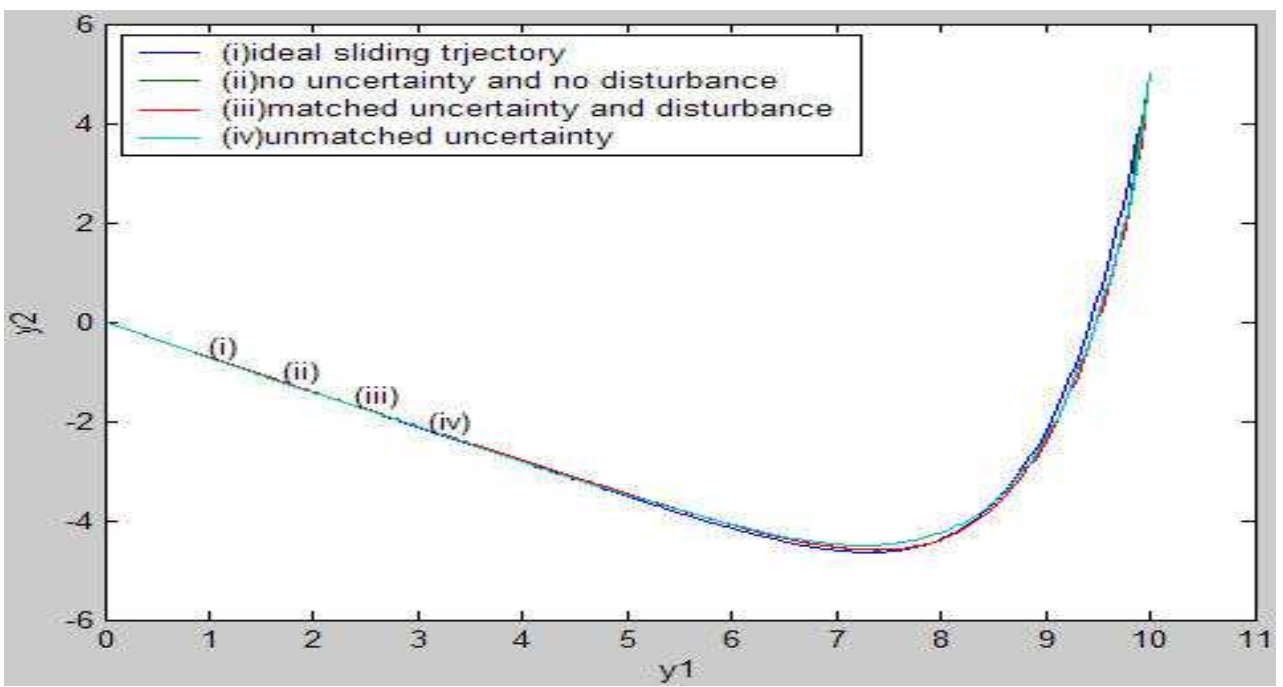

Fig. 14. Four phase trajectories (i)ideal sliding trajectory, (ii)no uncertainty and no disturbance (iii)matched uncertainty/disturbance, and (iv) unmatched uncertainty and matched disturbance by the continuously approximated input 


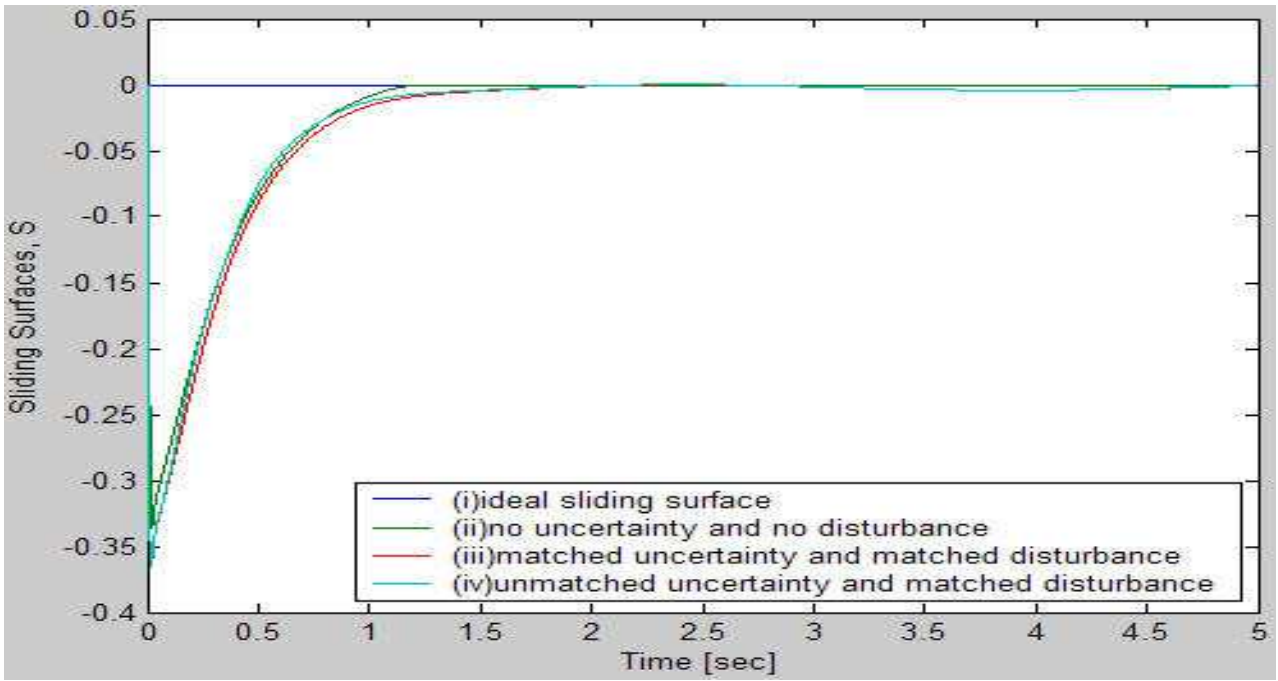

Fig. 15. Four sliding surfaces (i)ideal sliding surface, (ii)no uncertainty and no disturbance (iii)matched uncertainty/disturbance, and (iv) unmatched uncertainty and matched disturbance by the continuously approximated input

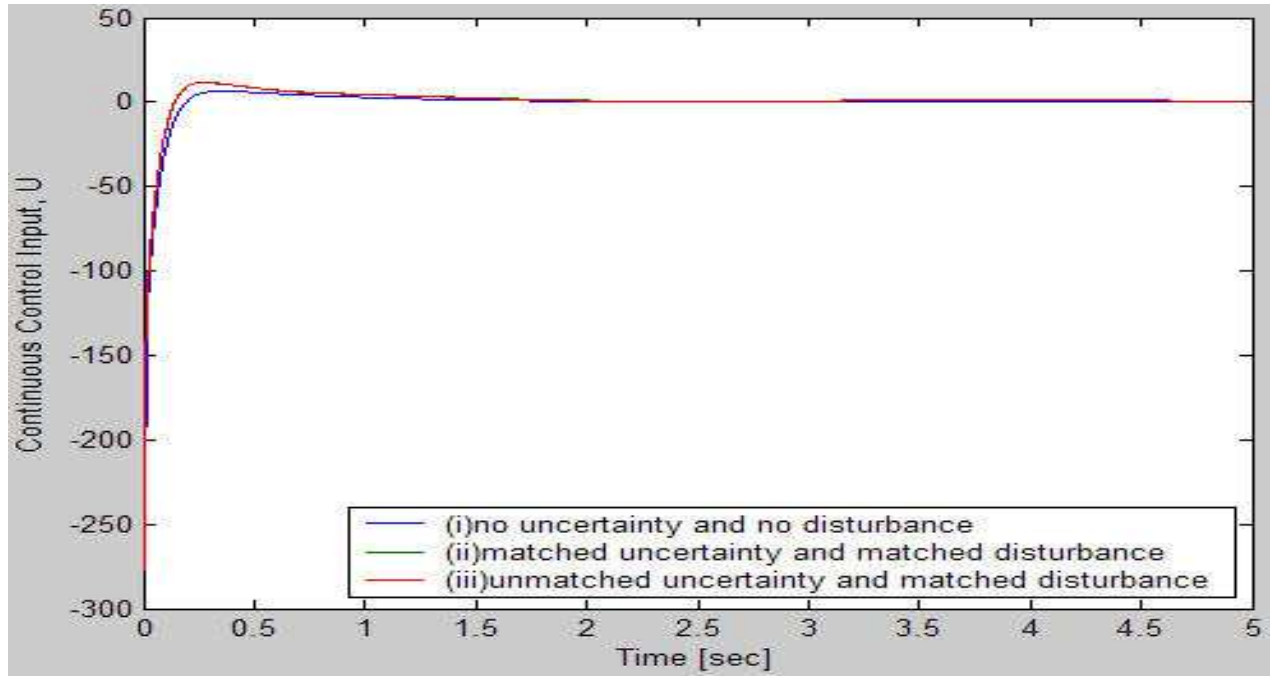

Fig. 16. Three case continuous control inputs $u_{0 c}$ (i)no uncertainty and no disturbance (ii)matched uncertainty/disturbance, and (iii) unmatched uncertainty and matched disturbance by the continuously approximated input for a positive $\delta_{0}=0.02$ 


\section{Conclusion}

In this chapter, a new practical robust full-state(output) feedback nonlinear integral variable structure controllers with the full-state(output) feedback integral sliding surfaces are presented based on state dependent nonlinear form for the control of uncertain more affine nonlinear systems with mismatched uncertainties and matched disturbance. After an affine uncertain nonlinear system is represented in the form of state dependent nonlinear system, a systematic design of the new robust integral nonlinear variable structure controllers with the full-state(output) feedback (transformed) integral sliding surfaces are suggested for removing the reaching phase. The corresponding (transformed) control inputs are proposed. The closed loop stabilities by the proposed control inputs with full-state(output) feedback integral sliding surface together with the existence condition of the sliding mode on the selected sliding surface are investigated in Theorem 1 and Theorem 2 for all mismatched uncertainties and matched disturbance. For practical application of the continuous discontinuous VSS, the continuous approximation being different from that of (Chern \& $\mathrm{Wu}, 1992)$ is suggested without severe performance degrade. The two practical algorithms, i.e., practical full-state feedback integral nonlinear variable structure controller with the fullstate feedback transformed input and the full-state feedback sliding surface and practical output feedback integral nonlinear variable structure controller with the output feedback input and the output feedback transformed sliding surface are proposed. The outputs by the proposed inputs with the suggested sliding surfaces are insensitive to only the matched uncertainty and disturbance. The unmatched uncertainties can influence on the ideal sliding dynamics, but the exponential stability is satisfied. The two main problems of the VSS, i.e., the reaching phase at the beginning and the chattering of the input are removed and solved.

\section{References}

Adamy, J. \& Flemming, A. (2004). Soft Variable Structure Control: a Survey. Automatica, vol.40, pp.1821-1844.

Anderson, B. D. O. \& More, J. B. (1990) Optimal Control, Prentice-Hall.

Bartolini, G. \& Ferrara, A. (1995). On Multi-Input Sliding Mode Control of Uncertain Nonlinear Systems. Proceeding of IEEE 34th CDC, p.2121-2124.

Bartolini, G., Pisano, A. \& Usai, E. (2001). Digital Second-Order Sliding Mode Control for Uncertain Nonlinear Systems. Automatica, vol.37 pp.1371-1377.

Cai, X., Lin, R., and Su, SU., (2008). Robust stabilization for a class of Nonlinear Systems. Proceeding of IEEE CDC pp.4840-4844, 2008.

Chen, W. H., Ballance, D. J. \& Gawthrop, P. J. (2003). Optimal Control of Nonlinear System:A Predictive Control Approach. Automatica, vol. 39, pp633-641.

Chern, T. L. \& Wu, Y. C., (1992). An Optimal Variable Structure Control with Integral Compensation for Electrohydraulic Position Servo Control Systems. IEEE T. Industrial Electronics, vol.39, no.5 pp460-463.

Decarlo, R. A., Zak, S. H., \& Mattews, G. P., (1988). Variable Structure Control of Nonlinear Multivariable Systems: A Tutorial. Proceeding of IEEE, Vol. 76, pp.212-232.

Drazenovic, B., (1969). The invariance conditions in variable structure systems, Automatica, Vol. 5, pp.287-295. 
Gutman, S. (1979). Uncertain dynamical Systems:A Lyapunov Min-Max Approach. IEEE Trans. Autom. Contr, Vol. AC-24, no. 1, pp.437-443.

Horowitz, I. (1991). Survey of Quantitative Feedback Theory(QFT). Int. J. Control, vol.53, no. 2 pp. $255-291$.

$\mathrm{Hu}$, X. \& Martin, C. (1999). Linear Reachability Versus Global Stabilization. IEEE Trans. Autom. Contr, AC-44, no. 6, pp.1303-1305.

Hunt, L. R., Su, R. \& Meyer, G. (1987). Global Transformations of Nonlinear Systems," IEEE Trans. Autom. Contr, Vol. AC-28, no. 1, pp.24-31.

Isidori, A., (1989). Nonlinear Control System(2e). Springer-Verlag.

Khalil, H. K. (1996). Nonlinear Systems(2e). Prentice-Hall.

Kokotovic, P. \& Arcak, M. (2001). Constructive Nonlinear Control: a Historical Perspective. Automatica, vol.37, pp.637-662.

Lee, J. H. \& Youn, M. J., (1994). An Integral-Augmented Optimal Variable Structure control for Uncertain dynamical SISO System, KIEE(The Korean Institute of Electrical Engineers), vol.43, no.8, pp.1333-1351.

Lee, J. H. (1995). Design of Integral-Augmented Optimal Variable Structure Controllers, Ph. D. dissertation, KAIST.

Lee, J. H., (2004). A New Improved Integral Variable Structure Controller for Uncertain Linear Systems. KIEE, vol.43, no.8, pp.1333-1351.

Lee, J. H., (2010a). A New Robust Variable Structure Controller for Uncertain Affine Nonlinear Systems with Mismatched Uncertainties," KIEE, vol.59, no.5, pp.945-949.

Lee, J. H., (2010b). A Poof of Utkin's Theorem for a MI Uncertain Linear Case," KIEE, vol.59, no.9, pp.1680-1685.

Lee, J. H., (2010c). A MIMO VSS with an Integral-Augmented Sliding Surface for Uncertain Multivariable Systems ," KIEE, vol.59, no.5, pp.950-960.

Lijun, L. \& Chengkand, X., (2008). Robust Backstepping Design of a Nonlinear Output Feedback System, Proceeding of IEEE CDC 2008, pp.5095-5099.

Lu, X. Y. \& Spurgeon, S. K. (1997). Robust Sliding Mode Control of Uncertain Nonlinear System. System \& control Letters, vol.32, pp.75-90.

Narendra, K. S. (1994). Parameter Adaptive Control-the End...or the Beginning? Proceeding of 33rd IEEE CDC.

Pan, Y. D. Kumar, K. D. Liu, G. J., \& Furuta, K. (2009). Design of Variable Structure Control system with Nonlinear Time Varying Sliding Sector. IEEE Trans. Autom. Contr, AC54, no. 8, pp.1981-1986.

Rugh, W. J. \& Shamma, J., (2000). Research on Gain Scheduling. Automatica, vol.36, pp.14011425.

Slottine, J. J. E. \& Li, W., (1991). Applied Nonlinear Control, Prentice-Hall.

Sun, Y. M. (2009). Linear Controllability Versus Global Controllability," IEEE Trans. Autom. Contr, AC-54, no. 7, pp.1693-1697.

Tang, G. Y., Dong, R., \& Gao, H. W. (2008). Optimal sliding Mode Control for Nonlinear System with Time Delay. Nonlinear Analysis: Hybrid Systems, vol.2, pp891-899.

Toledo, B. C., \& Linares, R. C., (1995). On Robust Regulation via Sliding Mode for Nonlinear Systems, System \& Control Letters, vol.24, pp.361-371.

Utkin, V. I. (1978). Sliding Modes and Their Application in Variable Structure Systems. Moscow, 1978. 
Vidyasagar, M. (1986). New Directions of Research in Nonlinear System Theory. Proc. of the IEEE, Vol.74, No.8, (1986), pp.1060-1091.

Wang, Y., Jiang, C., Zhou, D., \& Gao, F. (2007). Variable Structure Control for a Class of Nonlinear Systems with Mismatched Uncertainties. Applied Mathematics and Computation, pp.1-14.

Young, K.D., Utkin, V.I., \& Ozguner, U, (1996). A Control Engineer's Guide to Sliding Mode Control. Proceeding of 1996 IEEE Workshop on Variable Structure Systems, pp.1-14.

Zheng, Q. \& Wu, F. Lyapunov Redesign of Adpative Controllers for Polynomial Nonlinear systems," (2009). Proceeding of IEEE ACC 2009, pp.5144-5149. 


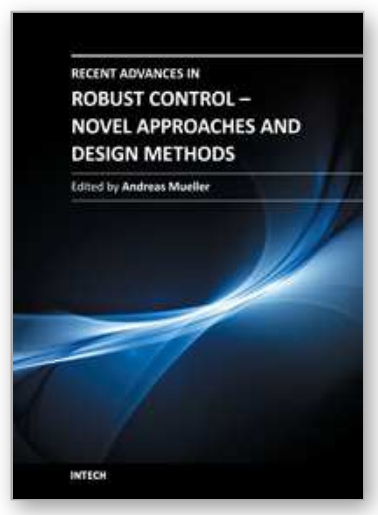

\author{
Recent Advances in Robust Control - Novel Approaches and \\ Design Methods \\ Edited by Dr. Andreas Mueller
}

ISBN 978-953-307-339-2

Hard cover, 462 pages

Publisher InTech

Published online 07, November, 2011

Published in print edition November, 2011

Robust control has been a topic of active research in the last three decades culminating in $\mathrm{H}$ 2/H_linfty and Imu design methods followed by research on parametric robustness, initially motivated by Kharitonov's theorem, the extension to non-linear time delay systems, and other more recent methods. The two volumes of Recent Advances in Robust Control give a selective overview of recent theoretical developments and present selected application examples. The volumes comprise 39 contributions covering various theoretical aspects as well as different application areas. The first volume covers selected problems in the theory of robust control and its application to robotic and electromechanical systems. The second volume is dedicated to special topics in robust control and problem specific solutions. Recent Advances in Robust Control will be a valuable reference for those interested in the recent theoretical advances and for researchers working in the broad field of robotics and mechatronics.

\title{
How to reference
}

In order to correctly reference this scholarly work, feel free to copy and paste the following:

Jung-Hoon Lee (2011). New Practical Integral Variable Structure Controllers for Uncertain Nonlinear Systems, Recent Advances in Robust Control - Novel Approaches and Design Methods, Dr. Andreas Mueller (Ed.), ISBN: 978-953-307-339-2, InTech, Available from: http://www.intechopen.com/books/recent-advances-inrobust-control-novel-approaches-and-design-methods/new-practical-integral-variable-structure-controllers-foruncertain-nonlinear-systems

\section{INTECH}

open science | open minds

\section{InTech Europe}

University Campus STeP Ri

Slavka Krautzeka 83/A

51000 Rijeka, Croatia

Phone: +385 (51) 770447

Fax: +385 (51) 686166

www.intechopen.com

\section{InTech China}

Unit 405, Office Block, Hotel Equatorial Shanghai

No.65, Yan An Road (West), Shanghai, 200040, China

中国上海市延安西路65号上海国际贵都大饭店办公楼 405 单元

Phone: +86-21-62489820

Fax: $+86-21-62489821$ 
(C) 2011 The Author(s). Licensee IntechOpen. This is an open access article distributed under the terms of the Creative Commons Attribution 3.0 License, which permits unrestricted use, distribution, and reproduction in any medium, provided the original work is properly cited. 